\title{
$8 \quad$ Programmable RNA base editing with a single gRNA-free enzyme
}

9
Wenjian Han ${ }^{1,2, \dagger}$, Wendi Huang ${ }^{1,2, \dagger}$, Miaowei Mao ${ }^{1, \dagger}$, Tong Wei ${ }^{1,2}$, Yanwen Ye ${ }^{1,2}$ \& Zefeng Wang ${ }^{1, *}$

1. Bio-Med Big Data Center, CAS Key Laboratory of Computational Biology, CAS Center for Excellence in Molecular Cell Science, Shanghai Institute of Nutrition and Health, University of Chinese Academy of Sciences, Chinese Academy of Sciences, Shanghai 200031, China

2. University of Chinese Academy of Sciences, Beijing 100049, China

*To whom correspondence should be addressed. Email: wangzefeng@picb.ac.cn

†The authors wish it to be known that, in their opinion, the first two authors should be regarded as Joint First Authors 
23 ABSTRACT

24 Programmable RNA editing enables rewriting gene expression without changing genome sequences.

25 Current tools for specific RNA editing dependent on the assembly of guide RNA into an RNA/protein

26 complex, causing delivery barrier and low editing efficiency. We report a new gRNA-free system, RNA

27 editing with individual RNA-binding enzyme (REWIRE), to perform precise base editing with a single

28 engineered protein. This artificial enzyme contains a human-originated programmable PUF domain to

29 specifically recognize RNAs and different deaminase domains to achieve efficient A-to-I or C-to-U

30 editing, which achieved $60-80 \%$ editing rate in human cells, with a few non-specific editing sites in the

31 targeted region and a low level off-target effect globally. The RNA-binding domain in REWIREs was

32 further optimized to improve editing efficiency and minimize off-target effects. We applied the

33 REWIREs to correct disease-associated mutations and achieve both types of base editing in mice. As

34 a single-component system originated from human proteins, REWIRE presents a precise and efficient

35 RNA editing platform with broad applicability. 


\section{INTRODUCTION}

38 Base editing is a new genetic modification strategy that directly installs point mutations into the DNAs 39 or RNAs without making pernicious breaks in nucleic acid chain (1-4). Directed base editing has 40 provided new potentials in treating genetic diseases caused by point mutations, such as Duchenne 41 muscular dystrophy (5) or Hutchinson-Gilford progeria syndrome (6). Compared to DNA manipulation, editing on RNAs is non-permanent and reversible, making it relative safer for in vivo application as a potential therapy (7-10). Previously several groups had used guided antisense RNA oligonucleotide to recruit adenosine deaminases from the ADAR (adenosine deaminases acting on $\underline{R} N A$ ) family to transform the RNA base from adenosine to inosine (recognized as guanosine) (3,11-16). However, the formation of dsRNA region and the recruitment of ADARs to the target RNAs are hard to control. Recently, Zhang's group developed two CRISPR-based systems using RNA-targeting Cas 13 protein and ADAR2 to achieve base editing of the specific RNAs: the REPAIR used the active domain of ADAR2 to achieve programmable adenosine to inosine (A-to-I) editing, and the RESCUE employed a modified ADAR2 to enable additional cytidine to uridine (C-to- $\mathrm{U})$ editing $(4,17)$. More recently, the Chi's group used similar design to engineer two CRISPR-based editing systems, REPAIRx and CURE, that improve the target recognition with dCasRx $(18,19)$. To overcome the difficulty for in vivo delivery of a large CRISPR-Cas system, a comparable but more concise system, CRISPR-Casinspired RNA targeting system (CIRTS), has also been developed by Dickinson's group to execute Ato-I editing on specific RNA targets (20).

All these engineered RNA base editing systems require the assembly of guide RNA (gRNA) and protein effector to form an RNA-protein complex for recognizing target RNAs. However, the cellular RNAs have much higher copy numbers than the genomic DNAs, the assembly and co-folding of the RNA-protein complex can be the rate-limiting step that reduce editing efficiency and hinder its application (21). Pairing of the gRNA and target RNA also leads to a relatively large editing window with potential "bystander" editing sites $(4,17,18)$. Finally, because Cas proteins are encoded by bacterial genome, the pre-existing immunity in a large fraction of human population presents a looming concern for future applications of CRISPR-based systems $(22,23)$. Alternatively, cellular RNAs can be directly manipulated using engineered proteins that contain a programmable RNAbinding module and different functional modules (24). For example, the RNA binding scaffold of 
67 to specifically bind any RNA base through interaction with the Watson-Crick edge (25-27). The PUF-

68 based systems with different functional domains have been developed by several groups to detect

69 cellular RNAs (28) or manipulate RNA splicing (29), translation (30), degradation (31) or

70 methylation/demethylation (32).

71 Here we engineered a single component RNA editing tool, $\underline{R} N A$ editing with individual $\underline{R} N A-$

72 binding enzyme (REWIRE), which contains a programmable PUF domain $(25,33)$ to specifically bind

73 RNAs and a functional domain from human ADARs to edit adenosine or APOBEC3A to edit cytidine

$74(2,34)$ (Figure S1A and S1B). Since the PUF domain can be reprogrammed to recognize almost any

75 short 8-nucleotide (8-nt) RNA sequences $(35,36)$, the REWIRE system can theoretically be designed

76 to install two key transitions (A-to-I or C-to-U) in any RNA. We further redesigned the PUF domain in

77 REWIREs to enhance the specificity and the editing efficiency. As a simple human-originated protein

78 without any RNA components, this system provides an easier and more practical system for basic

79 research and gene therapy.

81 MATERIAL AND METHODS

82 Plasmid construction

83 DNA fragments encoding the deaminase domains of the human ADAR1 and ADAR2 were 84 amplified from the cDNA of HEK 293T cells. The hyperactive variants of hADAR1/2 were generated with primers containing corresponding mutations $(37,38)$. All hADAR1/2 fragments were cloned into 86 the $\mathrm{pCl}-\mathrm{Neo}$ vector (Promega) to generate the $\mathrm{pCl}-\mathrm{ADAR}$ vectors. Different versions of codon optimized PUF repeats from human PUM1 that recognize all four RNA bases were chemically synthesized (GENEWIZ). The eight or ten PUF repeats were assembled by PCR amplification and inserted to N-terminal of ADARs to generate various Al-REWIRE expression vectors. The same strategy was applied to generate the CU-REWIRE expression vectors, where we first cloned human APOBEC3A gene into pCl-Neo and then inserted different versions of PUF domains. NLS and MTS peptide sequences were synthesized (GENEWIZ) and inserted into the CU-REWIRE 1.0 vector via

93 Gibson Assembly (New England Biolabs).

94 For the upgraded REWIREs, we assembled ten synthesized PUF repeats using PCR to generate 95 PUF-10R (35), and replaced the PUF-8R in the original REWIRE expression vectors, producing the 96 Al-REWIRE3.0, AI-REWIRE3.1 and CU-REWIRE2.0. For Al-REWIRE4.0, AI-REWIRE4.1 and CU- 
97 REWIRE3.0, we further modified the PUF-10R by inserting a short peptide loop (MNDGPHS) 98 between the ninth and the tenth repeat, forming PUF-10R*

99 For the wide type and mutant EGFP reporters, the coding sequences were amplified from pEGFP-

100 C1 (Clontech) with PCR and cloned into the pCDH-CMV-MCS-EF1-Puro vector (Promega). For the

101 reporters containing disease-relevant mutations, the coding sequences of target genes bearing pathogenic $\mathrm{G}>\mathrm{A}$ or $\mathrm{T}>\mathrm{C}$ mutations (as defined in ClinVar Database) were synthesized and cloned into the pCDH-CMV-MCS-EF1-Puro vector.

To clone various PUF domains that targeting different mRNAs into the REWIRE vectors, we used sequencing.

All targeted sites and primers were listed in the Table S1, and all sequences of REWIRE proteins were listed in the Supplementary Note 1.

\section{Mammalian cell culture and transfection}

HEK 293T (ATCC CRL-3216) and SH-SY5Y (ATCC CRL-2266) cells were maintained in Dulbecco's Modified Eagle's Medium with high glucose (HyClone) supplemented with $10 \%$ fetal bovine serum (FBS) at $37^{\circ} \mathrm{C}$ with $5 \% \mathrm{CO}_{2}$. $\mathrm{HCT} 116$ (ATCC CCL-247) cells were maintained in McCoy's 5a Medium (Gibco) supplemented with $10 \% \mathrm{FBS}$ at $37^{\circ} \mathrm{C}$ with $5 \% \mathrm{CO}_{2}$.

To assess the editing efficiency on the exogenous reporters, the cells were plated into a 12-well plate and transfected 12 hours later (approximate 70\% confluency) with different ratios of REWIRE expression vectors and reporter plasmids at a total DNA amount of $1 \mu \mathrm{g}$ per well. The resulting cells were harvested 48 hours after transfection for further analyses. To edit the endogenous mRNAs, HEK $293 T$ cells were transfected with $1 \mu \mathrm{g}$ REWIRE expression plasmids and harvested 48 hours post-

121 (Thermo Fisher Scientific) in 12-well plates (or 6-well plates with adjusted plasmid amount). To control for nonspecific transfection artifacts, we also treated the cells with only transfection reagent as a mock group.

\section{Flp-In cell line construction}

DNA fragment encoding dead EGFP (K163X) was amplified from pCDH-EGFP(K163X), and then inserted into modified pcDNA5/FRT/TO (without CMV enhancer) by Gibson Assembly. The resulting 
127 plasmid, pcDNA5/FRT/TO-EGFP(K163X), were co-transfected with pOG44 into Flp-In T-Rex 293

128 cells at a 1:9 ratio. 48 hours after the transfection, the cells were washed and split into fresh medium

129 ( $<25 \%$ confluency), further cultured at $37^{\circ} \mathrm{C}$ overnight. Subsequently the cells were feed with fresh

130 medium containing $100 \mu \mathrm{g} / \mathrm{ml} \mathrm{Hygromycin} \mathrm{every} 3$ days until the resistant colonies were visible. 3-4

131 individual colonies were picked for further use. The inserted sequences were also confirmed by

132 Sanger sequencing. The expression of the target gene could be induced by adding doxycycline to a

133 final concentration of $1 \mu \mathrm{g} / \mathrm{ml}$. After $24 \mathrm{~h}$ induction, the cells could be used to perform transfection and

134 then be collected 2 days later for measurement of RNA editing.

135

136

137

138

139

140

141

\section{Vector construction and production of AAV}

To construct the AAV vectors of REWIREs, the backbone plasmid pAV-FH vector (Vigenebio, AV88001) was linearized with Kpn1 and EcoRV, and the PCR-amplified AI-REWIRE 4.1 and CUREWIRE 3.0 were then cloned into linearized vector by Gibson Assembly. The resulting plasmid was named as pAV-AI-REWIRE 4.1 or pAV-CU-REWIRE 3.0. The AAV9 particles were produced by Vigenebio using HEK 293T cells using the triple-transfection protocols and purified with iodixanol gradient with standard protocols.

\section{Flow cytometry.}

To assess the correction of K163X mutation in EGFP, the transfected cells were analyzed using flow cytometry at indicated time point with CytoFLEX S (Beckman Coulter). The percent of EGFPpositive cells and fluorescence intensity were calculated to reflect editing efficiency. The data was analyzed and visualized by CytExpert software (Beckman Coulter).

\section{Western blot}

The HEK 293T cells were lysed in $1 \times$ SDS-PAGE loading buffer (Beyotime) and heated at $95^{\circ} \mathrm{C}$ for $10 \mathrm{~min}$. The mixtures were subsequently separated by $4-20 \%$ SDS-PAGE Gel (GenScript) and transferred to poly-vinylidene fluoride (PVDF) membrane. The primary antibodies, anti-FLAG antibody (F1084, Sigma-Aldrich) and anti-GAPDH antibody (14C10, CST), were used at a 1:2000 dilution as per manufacturer's instructions. For the secondary antibody, the HRP-linked goat antimouse IgG (CST\#7076) and goat anti-rabbit IgG (CST\#7074) were used at a 1:5000 dilution. The HRP conjugated secondary antibodies were visualized by using an enhanced chemiluminescence detection kit and ChemiDoc Touch Imaging System (Image Lab, Bio-Rad).

\section{RNA extraction and reverse transcription}


157

158

159

160

161

162

163

164

165

166

167

The RNAs were extracted using the TRIzol (Thermo Fisher) following the manufacturer's instructions. $1 \mu \mathrm{g}$ RNA was reverse transcribed using the PrimeScript RT reagent Kit with gDNA Eraser (Takara). The cDNA was then amplified for further analyses using deep sequencing on Illumina NextSeq platform. Extraction of genomic DNA was performed using the Mammalian Genomic DNA Extraction Kit (Cat\#D0061, Beyotime).

\section{Animal experiments}

All experiments with live animals were approved by the Institutional Animal Care and Use Committee (IACUC) of the Shanghai Institute of Nutrition and Health, Chinese Academy of Sciences. B6-EGFP mice (C57BL6-Tg (CAG-EGFP)10sb/J) were purchased from Jackson Laboratory. The male and female mice were evenly assigned to groups of predetermined sample size. AAV particles encoding Al-REWIRE 4.1 or CU-REWIRE 3.0 were injected into the B6-EGFP mice through tail intravenous injection $\left(2 \times 10^{12}\right.$ vector genomes per mouse in 4-week-old B6-EGFP mice $\left.(5,6)\right)$. Four weeks after injection, we collected muscles and heart samples from B6-EGFP mice and evaluated corresponding editing efficiency via RNA-Seq and Sanger-seq. No visible symptoms, such as a rough hair coat and moved slowly were observed in all mice after injection.

\section{Sanger sequencing and deep sequencing of RNA amplicons}

The cDNA was amplified with gene-specific primers flanking the target sequence (all primers and next-generation sequencing amplicons are listed in Table S1). The A-to-I or C-to-U editing rates were evaluated from Sanger sequencing using the percentage of peak area at the target adenosine site according to the sequencing chromatograms. For deep sequencing of RNA amplicons, the cDNA was amplified with gene-specific primers with appropriate forward and reverse adaptor sequences. The PCR products were purified, and the amplicon libraries were constructed using the 300-cycle Mi-Seq Reagent Kit v2 or Micro Kit v2 according to the manufacturer's protocols for paired-end sequencing (2×150bp) on the Illumina Hi-Seq X Ten machine by the Omics core facility of PICB. The RNA editing rates were evaluated by the percentage of the edited reads at the target sites (see below).

\section{RNA-seq experiments}

The cells were harvested 48 hours after transfection, and total RNAs were purified with TRIzol Reagent (Thermo Fisher). For each sample, $1 \mu \mathrm{g}$ of total RNAs were used to prepared ribo-minus RNA-seq libraries using the KAPA Stranded RNA-Seq Kit with RiboErase (Roche, KK8484), and the depletion of rRNAs confirmed by fluorometric quantification using the Qubit RNA HS Assay kit (Invitrogen). The sequencing was performed on an Illumina Hi-Seq X Ten machine by the Omics core 
188 facility of PICB $(2 \times 150$-base pair paired end; $50 \mathrm{M}$ reads for each sample). The summary of RNA189 seq data was listed in Table S2.

\section{RNA editing analysis using RNA-seq data}

191 For deep sequencing analysis, we first conducted quality control and trimming using TrimGalore

192 (0.6.0). To analyze sequences near the targeted sites, the sequence index was generated using the 193 targeted site sequence (upstream and downstream 200-nt) of each transcript. The RNA-seq reads were aligned to the index and quantified using BWA(0.7.17-r1188) (39). Alignment BAMs were then sorted by Samtools (1.9) (40), and the RNA editing sites were analyzed using REDItools (1.2) (41) with the following parameters: -t $20-p-u-m 20-T 6-6-W-v 1-n 0.0-e-d-I$. AWK scripts were used to filter out all the A-to-I or C-to-U conversions within the REWIRE targeting region, which were considered significant if the Fisher's exact test yielded a p-value less than 0.05 after multiple hypothesis correction by Benjamini Hochberg correction and at least 2 of 3 biological replicates identified the edit site. The mutations that appeared in both control and experimental groups were considered as single nucleotide polymorphisms.

To analyze entire transcriptome, AWK scripts were used to filter out the overexpressed REWIRE gene. After trimming, the reads longer than 90-nt were mapped to the reference genome (GRCh38/hg38 for human and GRCm38/mm10 for mouse) by STAR(2.4.2) (42). We then used Samtools (1.9) to sort alignment BAMs and remove duplicated reads by using Picard MarkDuplicates.jar(2.20.1-0). RNA base-editing variants were called using REDItools(1.2) with the following parameters: -t $20-p-P-u-U-a 6-6-A 6-6-v \quad 1-n$ consideration of strand information, all the A-to-I or C-to-U conversions were selected using AWK scripts. From all the called variants, downstream analyses focused solely on canonical chromosomes (1-22, $\mathrm{X}, \mathrm{Y}$ and $\mathrm{M}$ for human, 1-19, $\mathrm{X}, \mathrm{Y}$ and $\mathrm{M}$ for mouse).

An additional layer of filter for known variants was performed using SNPs data from dbSNP version 154 and Mouse Genome Project version 7 for human and mouse respectively. The variants shared by at least 2 out of 3 biological replicates in each sample were identified as the RNA editing sites. The RNA editing level of the mock group was treated as the background, and the global offtargets of REWIRE were calculated by subtracting the background variants. The thresholds we used to filter the SNVs are based on minimum coverage (20 reads), number of supporting reads (at least five mutated reads), allelic fraction $(\geq 0.5 \%)$, quality of the mapped reads $(>30)$, and base quality $(>30)$. 
218 For manhattan plot showing the distribution of edits across the transcriptome we set a depth cutoff at

219 100. The sequence motifs were generated with $R$ package ggseqlogo(0.1) (43). All plots were 220 generated with ggplot2 package in $\mathrm{R}$.

\section{Statistical analysis}

222 To assess whether REWIRE constructs perturbs natural editing homeostasis, we analyzed the 223 global editing sites shared by the mock group and PUF-Ctrl group. The differential RNA editing rates

224 at native A-to-I or C-to-U editing sites were assessed using Pearson's correlation coefficient analysis.

225 Pearson correlations of the editing rate between the mock group and PUF-Ctrl group were calculated 226 and annotated in Figure S2C and S2D. 


\section{RESULTS}

\section{Engineering Al-REWIREs for specific A-to-I transformation}

230 To engineer the artificial enzymes that specifically catalyze A-to-I editing, we fused the PUF domain

231 with the deaminase domain of ADAR1 or ADAR2 in different orders, generating PUF-ADAR and

232 ADAR-PUF fusion proteins (Figure S1C). We engineered the PUF domain to target EGFP (Enhanced

233 Green Fluorescent Protein) mRNA and co-expressed them in HEK293T cells, where the A-to-I editing

234 events were measured by sequencing the RT-PCR products. Only the PUF-ADAR configuration

235 showed detectable base editing activity near the PUF binding site (Figure S1C), and thus this configuration was used for the future design of all the AI-REWIREs (Figure 1A).

We engineered various versions of Al-REWIREs using wild type ADARs or their hyperactive mutants (ADAR1 in Al-REWIRE1.0, ADAR1-E1008Q in Al-REWIRE1.1, ADAR2 in Al-REWIRE2.0, ADAR2-E488Q in Al-REWIRE2.1) (37,38), and found that all Al-REWIREs showed efficient base editing at the on-targeted site A437 of the EGFP mRNA with highest editing rate at $\sim 81 \%$ (Figure $1 \mathrm{~B}$ and Figure S1D and S1E). In addition, the editing efficiency was also affected by the expression levels of AI-REWIREs, as judged by co-expression of AI-REWIREs and the target mRNAs in different ratios (Figure S1F). As expected, the editing sites occur at the upstream of AI-REWIRE binding region, consistent with that the PUF domain binds RNA in an antiparallel fashion (25). As a control, the expression of PUF or ADAR alone did not induce detectable editing near the targeted region (Figure 1B). The estimated editing rates are highly correlated between the measurements with Sanger sequencing and RNA-seq (Figure S2A and S2B). While the Sanger sequencing provides a more accurate picture at the targeted site and the bystander editing site near the REWIRE binding window, the RNA-seq can give a global picture for the transcriptome-wide off-target effect.

Transcriptome-wide RNA-seq revealed that the major off-target A-to-I editing sites by AlREWIREs are in the targeted EGFP mRNA rather than endogenous RNAs (Figure 1C, blue vs. grey). As expected, the AI-REWIRE1.1 and AI-REWIRE2.1 have higher editing activities at both the target site and the off-target sites compared to those with wild-type domains (version 1.0 and 2.0) (Figure $1 \mathrm{C}$ and 1D). In addition, we found no significant difference in global editing of endogenous sites between cells transfected with PUF domain only and mock vector (Figure S2C and S2D), suggesting PUF domain had little impact on normal editing by endogenous ADARs. Consistent with sequence preferences of ADARs (44), the AI-REWIREs were found to have a slight preference of guanine next to the edited adenosines (Figure 1E).

To determine the optimal distance between the PUF binding site and the editing site, we constructed several Al-REWIREs that specifically bind to the shifting positions at downstream of a natural edited site in GRIA2 mRNA (Figure 1F and Figure S3A). We observed an optimal editing window between the position 7 to 9 from the PUF binding site to the on-target editing site and with a low bystander editing (Figure 1F). When the binding sites of Al-REWIREs were shifted toward 3' end, editing of a downstream adenosine (A1824) was increased (Figure 1F and Figure S3A). Our subsequent results suggest that Al-REWIREs had a fairly narrow window for optimal editing ( $3 \mathrm{nt})$

266 (Figure S3B and S3C). 


\section{Application of Al-REWIREs in base correction}

We next applied this system to repair a nonsense mutation (K163X) in EGFP gene. Initially the dead EGFP was co-expressed with two versions of Al-REWIREs designed to recognize a downstream sequence CUUCAAGA, and we found that the adenosine was efficiently edited to restore the green fluorescence (Figure S4A), enabling a functional rescue of EGFP in $40-60 \%$ transfected cells as judged by flow cytometry (Figure S4B). Furthermore, we generated a stable cell line containing a single copy of dead EGFP stably integrated into the genome by Flp-In system (Figure 2A). The dead EGFP was expressed from a moderate CMV promoter without the proximal enhancer to mimic the expression level of a typical endogenous gene. We expressed the two versions of Al-REWIREs in different amounts (Figure 2B), and found a dose-dependent base editing of K163X site, reaching $20 \%$ editing rate with the Al-REWIRE2.1 (Figure 2C-2E). We also monitored the phenotypic change for several days after AI-REWIRE transfection and found that the EGFP signals appeared at day one after transfection, reached the peak at day 2, and faded out after day 4 (Figure 2F).

We further designed Al-REWIREs to edited several endogenous genes, including the cancerassociated TP53 gene that was previously edited by other RNA editing tools $(4,16)$. Using different amounts of plasmids in transfection, we again found a dose-dependent editing of the A553 in TP53 gene with efficiency ranging from $13 \%$ to $58 \%$ with two versions of Al-REWIREs (Figure $2 \mathrm{G}$ and $2 \mathrm{H}$ ). For the five endogenous editing sites tested, we achieved $15-59 \%$ editing using different AI-REWIREs (Figure $2 \mathrm{~J}$ and Figure $\mathrm{S} 4 \mathrm{C}$ ), suggesting that AI-REWIREs are capable of editing endogenous mRNAs although the editing efficiency remains to be improved.

We further examined whether Al-REWIREs could effectively edit disease-related mutations in human cells. Different versions of AI-REWIREs were engineered to target several mutations, including two DMD mutations that cause Duchenne muscular dystrophy. We co-expressed the mutated genes with corresponding AI-REWIREs, and found that the mutated base can be corrected efficiently, with AI-REWIREs yielding higher editing rates ranging from $20 \%$ to $60 \%$ (Figure $2 \mathrm{I}-2 \mathrm{~J}$ and Figure S4D-4E).

\section{Engineering CU-REWIREs for specific C-to-U conversion}

The successful engineering of AI-REWIREs prompted us to employ similar design for C-to-U editing. We combined the PUF domain and the cytosine deaminase APOBEC3A (apolipoprotein B mRNA editing enzyme catalytic subunit $3 A$, or $A 3 A$ ) with a peptide linker to generate the CU-REWIRE (Figure $3 A$ ). As a proof of concept, we tested the activity of CU-REWIREs on EGFP mRNA and found that the CU-REWIRE could enable precise $\mathrm{C}$-to-U editing with efficiency up to $\sim 60 \%$ (Figure $3 \mathrm{~B}$ and Figure S5A). Additionally, the CU-REWIRE(Mut) containing an inactive A3A mutation (E72A) did not show any detectable editing (45), suggesting that the base editing is indeed catalyzed by A3A (Figure 3B).

We further used RNA-seq to examine the off-target C-to-U editing induced by CU-REWIRE, and found some off-target editing sites in both the targeted EGFP mRNA and other endogenous 
306 transcripts (Figure 3C). Interestingly, the endogenous off-target sites with high editing rates are mostly

307 expressed in a very low level, which might be contributed by an "editing noise" (see discussion for 308 more details). Compared to AI-REWIREs, the CU-REWIRE appeared to have a tighter editing window 309 and performed C-to-U editing at position 2 after the PUF binding site (Figure 3D). Moreover, the 310 edited cytidines were predominantly found within the short consensus motif UC (Figure 3E). Such 311 preference was experimentally validated using all base combinations around the targeted cytidine, 312 where the UCN sequence was strongly edited by the CU-REWIRE and the CCN was also tolerated to 313 a less extend (Figure 3F). This finding is consistent with the target preference of APOBEC3A (45). 314 Interestingly, we found that the guanosine at immediate downstream of the cytidine can reduce editing efficiency, reflecting an unreported feature for APOBEC3A target preference (Figure 3F).

To test if CU-REWIREs are capable of editing endogenous mRNAs, we designed a CU-REWIRE to target an endogenous gene CTNNB1, and achieved $20-45 \%$ editing efficiency at the targeted C1549 site in a dose-dependent fashion (Figure $3 \mathrm{G}$ and $3 \mathrm{H}$ ). To further test the general applicability in RNA base correction, we designed CU-REWIREs targeting two disease-related $\mathrm{T}>\mathrm{C}$ mutations in human genes (EZH2 in Weaver syndrome, SCN1A in Dravet syndrome). We found that the deleterious mutations were indeed corrected via cytidine editing with efficiency ranging from $21 \%$ to $29 \%$ (Figure S5B and S5C). Furthermore, we also targeted the C246 in SARS-Cov-2 membrane protein, and achieved high editing efficiency ( 62\%) (Figure S5D).

\section{Improvement of the REWIREs}

To optimize the REWIREs, we redesigned a PUF domain with 10 tandem repeats (PUF_10R) that can specifically recognize RNAs by a 10-nt binding site rather than an 8-nt site of wild type PUF_8R, thus should significantly increase its specificity and reduce off-targets $(27,35)$. We applied this design strategy to generate AI-REWIRE3.0 and AI-REWIRE4.0 using two versions of PUF_10R (Figure 4A, see methods), and further engineered AI-REWIRE3.1 and AI-REWIRE4.1 by upgrading deaminase domain with hyperactive ADAR2. As expected, the Al-REWIREs with PUF_10R substantially reduced both the editing rates and the numbers of off-target sites without sacrificing the on-target editing efficiency (Figure 4B and 4C). Compared to the most active Al-REWIRE2.1 (on-target editing rate $=81 \%$, off-target counts $=31804$ ), the updated Al-REWIRE3.1 had much less off-target counts (1908) and a decent editing rate ( $62 \%)$ while the AI-REWIRE4.1 showed similar tendency (on-target editing rate $=72 \%$, off-target counts $=6664$ ) (Figure $4 \mathrm{C}$ and $4 \mathrm{G}$ ). Interestingly, such improvement was achieved with a lower expression level than the early versions (Figure S6A and S6B), which is a desirable feature for in vivo application where the high expression of engineered editase may be difficult to achieve and/or have adverse effects. We further used the upgraded AI-REWIRE4.0 to edit an endogenous gene (PPIB), and found that it achieved a comparable editing rate to the AlREWIRE2.0 targeting the same site (Figure S6C), but with much less off-targeting effect (Figure S6D), which is consistent with its longer recognition sequence (10nt vs. 8nt). Since the PUF domain recognizes a short RNA sequence, this specific Al-REWIREs has other recognition sites in the 
345 limitation of all engineered factors using PUF domain, which could be managed through target design

346 and additional controls in protein expression (see discussion). As expected, both Al-REWIREs

347 function in a dose-dependent manner in editing endogenous targets (Figure S6E).

With the same strategy, we upgraded the original CU-REWIRE (CU-REWIRE1.0) into CUREWIRE2.0 and CU-REWIRE3.0 using different versions of PUF_10R (Figure 4D and 4E), and measured their editing efficiency on EGFP mRNA (Figure 4E and Figure S6B). While all the CUREWIREs showed precise editing at the targeted cytidine (C459), the CU-REWIRE3.0 achieved the highest editing rate (up to $65 \%$ ) and relatively low off-target effects across the entire transcriptome (Figure $4 \mathrm{~F}$ and $4 \mathrm{G}$ ), indicating a comprehensive improvement. We designed new CU-REWIRE3.0s targeting two disease-related $\mathrm{T}>\mathrm{C}$ mutations in EZH2 and SCN1A genes, and found that these mutations were indeed corrected via cytidine editing with modestly increased efficiency while the target specificity was improved (Figure S6F and S6G).

To take advantage of REWIREs that can introduce multiple variants in RNAs, we applied AIREWIRE and CU-REWIRE together for concurrent editing on a single transcript, producing efficient Ato-I and C-to-U editing simultaneously (Figure $4 \mathrm{H}$ and Figure S7). We also noticed that using two REWIREs in combination showed comparable editing rates to the AI-REWIRE-4.1 or CU-REWIRE3.0 alone at the single base (compare Figure $4 \mathrm{H}$ to Figure 4B and $4 \mathrm{E}$ ), suggesting they may function independently. Importantly, the overlap of the binding sites for two editases did not interfere with the concurrent editing (Figure 4H), suggesting they undergo dynamic turn over on the target RNA like what would be expected for bona fide enzymes. Within each targeted mRNA molecule, both AlREWIRE 4.1 and CU-REWIRE3.0 preferably edited on the single intended site as judged by reanalyzing the RNA-seq data (Figure S8). In addition, we also tested the REWIREs in additional two human cell lines (SH-SY5Y and HCT116), and found that different versions of REWIREs achieved comparable editing efficiency on the targeted sites $(60 \%-86 \%$ for A-to-I editing, and $25-80 \%$ for C-toU, Figure S9).

\section{RNA editing in mouse model with REWIREs}

372 We further tested the in vivo editing efficacy of the REWIRE system using a transgenic mouse model 373 (B6-EGFP) that stably expresses EGFP. The EGFP-targeting Al-REWIRE4.1 or CU-REWIRE3.0 374 were packed in AAV9 vectors, which were systematically delivered to 4-week-old mice using intravenous injections at the tail vein (Figure 5A and $5 \mathrm{C}$ ). Because the AAV9 vectors has a high tropism for muscle and heart $(46,47)$, we collected the muscle and heart samples at 4 weeks postinjection, and evaluated the editing efficiency via Sanger sequencing and RNA-seq. At the tibialis anterior muscle of the three mice, the AI-REWIRE 4.1 achieved $27 \%-34 \%$ editing efficiency at the targeted A437 site of EGFP (Figure 5B), whereas the CU-REWIRE 3.0 targeting the C459 of the EGFP showed $44 \%-51 \%$ editing efficiency (Figure 5D). As a control, the expression of the PUF domain alone through same AAV9 vectors did not induce detectable editing (Figure 5B and 5D). 
We further measured the low off-target editing on the mouse transcriptome using RNA-seq profiling, and found that most off-target sites were edited with low efficiency (Figure 5E and 5F). In addition, we observed effective RNA editing of the intended sites in both heart and muscles, which is consistent with the tissue preference of AAV9 (Figure $5 \mathrm{G}$ ). These results suggested that REWIREs can effectively edit RNAs in animal, however the editing efficiency remains to be optimized.

\section{DISCUSSION}

Compared to DNA, targeted RNA editing is reversible and more flexible, presenting a more tractable route for gene manipulation. Here we engineered a series of programmable RNA editases for specific base editing. With different functional domains, the REWIREs can effectively achieve both A-to-I and C-to- $U$ editing, and can be used in correcting pathogenic mutations of endogenous genes. The protein sequences of this system are entirely originated from human proteome, which should avoid the innate immunity from CRISPR-based system using bacterial proteins. Importantly, the REWIRE system achieved in vivo base editing in mice through systematic delivery with AAV (Figure 5), suggesting its potential in personalized gene therapy.

The REWIREs consist of a single protein that recognizes target RNAs without the assembly of gRNA/protein complex, thus harboring high efficiency while avoiding assembly intermediates or misassembled by-products. The RNA-binding affinity is similar for all PUF_8Rs with similar GC content in the targets $\left(K_{d}\right.$ in the order of $\left.\mathrm{nM}\right)(25,29)$, and PUF_10R can increase the binding affinity by $\sim 10$ fold (35). A single component editase can also be transported to different subcellular compartments (e.g., mitochondria or chloroplast), which may be used in specific editing of extranuclear genes. As an RNA editing tool, the optimized REWIRE has several desirable features. First, the REWIREs with PUF_10R have a small size (873 aa and 644aa for Al- and CU- REWIREs) suitable for AAV delivery in clinical applications. Second, the REWIRE sequences are entirely originated from human genes, making it less likely to induce immune response for in vivo applications. Third, the REWIRE directly converts the RNA base without using endogenous repair pathways, thus may be able to edit RNA in post-mitotic cells including neurons. Finally, different REWIREs can be independently applied to achieve simultaneous A-to-I and C-to-U editing in the same transcript, allowing concurrent editing of multiple mutations that synergistically cause human diseases (Figure S8).

As a new technology, the REWIRE system also has limitations that should be improved with future optimization. First, PUF domains target a relatively short sequence (8 to 10-nt in this study), which is shorter than the CRISPR-based system but is higher than the 7-nt seed match of siRNAs. This may contribute to some unintended targets with similar recognition sites in the transcriptome. This problem could be managed by optimizing target designs. For example, the specificity could be improved by using tandem PUFs or a PUF with even more repeats (a PUF with 16 repeats has been proved feasible (35)); and computational analyses could be applied during target selection to minimize unwanted targets in transcriptome. Second, REWIREs still have bystander editing sites within the editing window, although this problem is less severe than the CRISPR-based editing tools $(4,17)$. It is possible to optimize the deaminase domain and the linker region to increase editing 
422 accuracy. Theoretically, we should be able to introduce mutations in the deaminase domain to

423 increase the selectivity of editing sites, and/or modify the linker region to refine the editing window and 424 reduce the bystander editing.

425 The off-target effect has been a major concern for gene editing, especially for the genomic DNA 426 because the deleterious changes will be permanent. However, the off-target editing on RNAs may be 427 less detrimental since the cellular RNAs constantly undergo synthesis and degradation cycles. Both 428 the edited and unedited versions of transcripts are co-expressed in the same cells, probably damping 429 the side effect of the off-targeting editing. The off-target effects are mainly depended on the choice of 430 functional domains. For example, the APOBEC1 were shown to induce more off-target editing sites in 431 both DNA and RNA than the APOBEC3A, which can be reduced by more specific deaminase (7432 10,48). We found that REWIREs with ADAR2 showed less off-target effects than ADAR1, and the 433 hyperactive mutants increased off-target effects while improving editing rates (Figure 1C and 1D). 434 Further optimization on the PUF domain also remarkably reduced the off-target effects while 435 maintaining the high editing efficiency (Figure 4).

436 In addition, the common procedures to measure editing sites using RNA-seq tend to over437 estimate off-target sites, probably due to the sequencing errors associated with NGS technologies 438 and underlying genomic variants that may confound the analyses (49). It has been reported that the 439 accuracy for quantifying the RNA editing events can be generally improved by a higher sequencing 440 depth and more uniform read coverage (50). Therefore, for a more reliable estimation, we sequenced 441 the total RNAs (rather than mRNAs) with a deep coverage ( 50 million 150 bp paired-end reads for 442 each sample) and conducted technical triplicates to reduce sequencing errors. We found that many 443 "editing" sites covered only by a small number of reads have surprisingly high editing rates. However, 444 such estimation may not be reliable, as increasing the cut-off for the read numbers will eliminate these 445 "editing" sites whereas the true positive editing events remain unchanged (Figure S10). This "editing 446 noise" is similar to a previous report that virtually all adenosines within human Alu regions could be 447 edited as judged by an ultra-deep sequencing of selected Alu elements (>5000 reads/site) (51).

448 Compared to other gRNA-based RNA editing tools, including the new CRISPR-based REPAIRv2 449 (4) and REPAIRx (18) systems, the AI-REWIREs showed a higher editing efficiency at the targeted 450 sites and a slightly higher off-target effect, the CU-REWIREs presented comparable properties on 451 editing efficiency and off-target effect to the latest C-to-U editing tools, CURE systems in HEK 293T 452 cells (19). This is a rough comparison because of the technical challenges in estimating off-target 453 effect (Figure S10). Nevertheless, REWIRE proteins present a simple and human originated system 454 with comparable performance. Multiple technologies may complement to each other in editing 455 different targets, where the users should consider a tradeoff between editing efficiency and the off456 target effects to find a sweet spot.

457 Although we have improved the REWIREs with different versions of PUFs, additional research is 458 certainly needed to further optimize this system. Compared to previous tools using adenosine 459 deaminase domain of ADARs for A-to-I editing and modified ADAR2 for C-to-U editing (4)'(17), we 460 achieved C-to-U editing with cytidine deaminase Apobec3A that is smaller than ADARs. The 461 APOBAC family has many members with various specificities and strengths, therefore further 
exploration on the APOBAC family with possible modifications may expand the functional modules of REWIREs to edit additional bases. In addition, adjusting the composition and length of the internal linker in REWIRE may also improve its performance. Collectively, the REWIRE represents a simple and practical alternative to CRISPR-based approaches, expanding the current RNA editing toolbox.

\section{AVAILABILITY}

The original RNA and DNA sequencing datasets have been deposited in the NCBI Gene Expression Omnibus (accession code GSE155734) and the National Omics Data Encyclopedia (accession code OEP001013) for public downloading. Custom computer code is available upon request.

\section{SUPPLEMENTARY DATA}

Supplementary Data are available at NAR online.

\section{ACKNOWLEDGEMENT}

We thank Ms. Yun Jiang for helps in preparing the paper, and members of Wang lab for discussion and comments of this manuscript. We thank Dr. Li Yang for the generous gift of human APOBEC3A.

\section{FUNDING}

This work is supported by Science and Technology Commission of Shanghai Municipality (17JC1404900 and 18XD1404400 to Z.W.), National Natural Science Foundation of China (31730110, 31570823, 91940303 and 31661143031 to Z.W., 31971367 to M.M.), the National Key Research and Development Program of China (2018YFA0107602) and the Strategic Priority Research Program of Chinese Academy of Sciences (XDB38040100 to Z.W.). M.M. is supported by the National Postdoctoral Program for Innovative Talents (BX20180336) and Shanghai Super Postdoctoral Program. Z.W. is supported by the CAS Pioneer Hundred Talents program (type A). Funding for open access charge: National Natural Science Foundation of China.

\section{CONFLICT OF INTEREST}

The authors declare that a patent application related to this technology has been filed. Z.W. is a founder and scientific adviser for Enzerna Biosciences Inc that seeks commercial applications for engineered PUF factors. 
bioRxiv preprint doi: https://doi.org/10.1101/2021.08.31.458316; this version posted August 31, 2021. The copyright holder for this preprint (which was not certified by peer review) is the author/funder, who has granted bioRxiv a license to display the preprint in perpetuity. It is made available under aCC-BY-NC-ND 4.0 International license. 
500

501

502

503

504

505

506

507

508

509

510

511

512

513

514

515

516

517

518

519

520

521

522

523

524

525

526

527

528

529

530

531

532

533

534

535

536

537

538

539

540

541

542

543

544

545

546

547

\section{REFERENCES}

1. Komor, A.C., Kim, Y.B., Packer, M.S., Zuris, J.A. and Liu, D.R. (2016) Programmable editing of a target base in genomic DNA without double-stranded DNA cleavage. Nature, 533, 420-424.

2. Wang, X., Li, J., Wang, Y., Yang, B., Wei, J., Wu, J., Wang, R., Huang, X., Chen, J. and Yang, L. (2018) Efficient base editing in methylated regions with a human APOBEC3A-Cas9 fusion. Nat Biotechnol, 36, 946-949.

3. Stafforst, T. and Schneider, M.F. (2012) An RNA-deaminase conjugate selectively repairs point mutations. Angew Chem Int Ed Engl, 51, 11166-11169.

4. Cox, D.B.T., Gootenberg, J.S., Abudayyeh, O.O., Franklin, B., Kellner, M.J., Joung, J. and Zhang, F. (2017) RNA editing with CRISPR-Cas13. Science, 358, 1019-1027.

5. Katrekar, D., Chen, G., Meluzzi, D., Ganesh, A., Worlikar, A., Shih, Y.R., Varghese, S. and Mali, P. (2019) In vivo RNA editing of point mutations via RNA-guided adenosine deaminases. Nat Methods, 16, 239-242.

6. Koblan, L.W., Erdos, M.R., Wilson, C., Cabral, W.A., Levy, J.M., Xiong, Z.M., Tavarez, U.L., Davison, L.M., Gete, Y.G., Mao, X. et al. (2021) In vivo base editing rescues Hutchinson-Gilford progeria syndrome in mice. Nature, 589, 608-614.

7. Erwei Zuo, Y.S., Wu Wei, Tanglong Yuan, Wenqin Ying, Hao Sun, Liyun Yuan4, Lars M. Steinmetz, Yixue Li, Hui Yang. (2019) Cytosine base editor generates substantial off-target single nucleotide variants in mouse embryos. science, 289-292.

8. Zhou, C., Sun, Y., Yan, R., Liu, Y., Zuo, E., Gu, C., Han, L., Wei, Y., Hu, X., Zeng, R. et al. (2019) Off-target RNA mutation induced by DNA base editing and its elimination by mutagenesis. Nature, $\mathbf{5 7 1}, \mathbf{2 7 5 - 2 7 8 . ~}$

9. Grunewald, J., Zhou, R., Garcia, S.P., lyer, S., Lareau, C.A., Aryee, M.J. and Joung, J.K. (2019) Transcriptome-wide off-target RNA editing induced by CRISPR-guided DNA base editors. Nature, 569, 433-437.

10. Kim, D., Lim, K., Kim, S.T., Yoon, S.H., Kim, K., Ryu, S.M. and Kim, J.S. (2017) Genome-wide target specificities of CRISPR RNA-guided programmable deaminases. Nat Biotechnol, 35, 475-480.

11. Montiel-Gonzalez, M.F., Vallecillo-Viejo, I., Yudowski, G.A. and Rosenthal, J.J. (2013) Correction of mutations within the cystic fibrosis transmembrane conductance regulator by site-directed RNA editing. Proc Natl Acad Sci U S A, 110, 18285-18290.

12. Fukuda, M., Umeno, H., Nose, K., Nishitarumizu, A., Noguchi, R. and Nakagawa, H. (2017) Construction of a guide-RNA for site-directed RNA mutagenesis utilising intracellular A-to-I RNA editing. Sci Rep, 7, 41478.

13. Wettengel, J., Reautschnig, P., Geisler, S., Kahle, P.J. and Stafforst, T. (2017) Harnessing human ADAR2 for RNA repair - Recoding a PINK1 mutation rescues mitophagy. Nucleic Acids Res, 45, 2797-2808.

14. Vogel, P., Moschref, M., Li, Q., Merkle, T., Selvasaravanan, K.D., Li, J.B. and Stafforst, T. (2018) Efficient and precise editing of endogenous transcripts with SNAP-tagged ADARs. Nat Methods, 15, 535-538.

15. Merkle, T., Merz, S., Reautschnig, P., Blaha, A., Li, Q., Vogel, P., Wettengel, J., Li, J.B. and Stafforst, T. (2019) Precise RNA editing by recruiting endogenous ADARs with antisense oligonucleotides. Nat Biotechnol, 37, 133-138.

16. Qu, L., Yi, Z., Zhu, S., Wang, C., Cao, Z., Zhou, Z., Yuan, P., Yu, Y., Tian, F., Liu, Z. et al. (2019) Programmable RNA editing by recruiting endogenous ADAR using engineered RNAs. Nat Biotechnol, 37, 1059-1069.

17. Abudayyeh, O.O., Gootenberg, J.S., Franklin, B., Koob, J., Kellner, M.J., Ladha, A., Joung, J., Kirchgatterer, P., Cox, D.B.T. and Zhang, F. (2019) A cytosine deaminase for programmable single-base RNA editing. Science, 365, 382-386.

18. Liu, Y., Mao, S., Huang, S., Li, Y., Chen, Y., Di, M., Huang, X., Lv, J., Wang, X., Ge, J. et al. (2020) REPAIRx, a specific yet highly efficient programmable A > I RNA base editor. EMBO J, 39, e104748. 
548

549

550

551

552

553

554

555

556

557

558

559

560

561

562

563

564

565

566

567

568

569

570

571

572

573

574

575

576

577

578

579

580

581

582

583

584

585

586

587

588

589

590

591

592

593

594

595

596

597

598

599

600

601

19. Huang, X., Lv, J., Li, Y., Mao, S., Li, Z., Jing, Z., Sun, Y., Zhang, X., Shen, S., Wang, $X$. et al. (2020) Programmable C-to-U RNA editing using the human APOBEC3A deaminase. EMBO J, 39, e104741.

20. Rauch, S., He, E., Srienc, M., Zhou, H., Zhang, Z. and Dickinson, B.C. (2019) Programmable RNA-Guided RNA Effector Proteins Built from Human Parts. Cell, 178, 122-134 e112.

21. Mekler, V., Minakhin, L., Semenova, E., Kuznedelov, K. and Severinov, K. (2016) Kinetics of the CRISPR-Cas9 effector complex assembly and the role of 3 '-terminal segment of guide RNA. Nucleic Acids Res, 44, 2837-2845.

22. Gough, V. and Gersbach, C.A. (2020) Immunity to Cas9 as an Obstacle to Persistent Genome Editing. Mol Ther, 28, 1389-1391.

23. Charlesworth, C.T., Deshpande, P.S., Dever, D.P., Camarena, J., Lemgart, V.T., Cromer, M.K., Vakulskas, C.A., Collingwood, M.A., Zhang, L., Bode, N.M. et al. (2019) Identification of preexisting adaptive immunity to Cas9 proteins in humans. Nat Med, 25, 249-254.

24. Wei, H. and Wang, Z. (2015) Engineering RNA-binding proteins with diverse activities. Wiley Interdiscip Rev RNA, 6, 597-613.

25. Cheong, C.G. and Hall, T.M. (2006) Engineering RNA sequence specificity of Pumilio repeats. Proc Natl Acad Sci U S A, 103, 13635-13639.

26. Dong, S., Wang, Y., Cassidy-Amstutz, C., Lu, G., Bigler, R., Jezyk, M.R., Li, C., Hall, T.M. and Wang, Z. (2011) Specific and modular binding code for cytosine recognition in Pumilio/FBF (PUF) RNA-binding domains. J Biol Chem, 286, 26732-26742.

27. Filipovska, A., Razif, M.F., Nygard, K.K. and Rackham, O. (2011) A universal code for RNA recognition by PUF proteins. Nat Chem Biol, 7, 425-427.

28. Ozawa, T., Natori, Y., Sato, M. and Umezawa, Y. (2007) Imaging dynamics of endogenous mitochondrial RNA in single living cells. Nat Methods, 4, 413-419.

29. Wang, Y., Cheong, C.G., Hall, T.M. and Wang, Z. (2009) Engineering splicing factors with designed specificities. Nat Methods, 6, 825-830.

30. Cooke, A., Prigge, A., Opperman, L. and Wickens, M. (2011) Targeted translational regulation using the PUF protein family scaffold. Proc Natl Acad Sci U S A, 108, $15870-15875$.

31. Choudhury, R., Tsai, Y.S., Dominguez, D., Wang, Y. and Wang, Z. (2012) Engineering RNA endonucleases with customized sequence specificities. Nat Commun, 3, 1147.

32. Shinoda, K., Suda, A., Otonari, K., Futaki, S. and Imanishi, M. (2020) Programmable RNA methylation and demethylation using PUF RNA binding proteins. Chem Commun (Camb), 56, 1365-1368.

33. Adamala, K.P., Martin-Alarcon, D.A. and Boyden, E.S. (2016) Programmable RNAbinding protein composed of repeats of a single modular unit. Proc Natl Acad Sci U S A, 113, E2579-2588.

34. Salter, J.D., Bennett, R.P. and Smith, H.C. (2016) The APOBEC Protein Family: United by Structure, Divergent in Function. Trends Biochem Sci, 41, 578-594.

35. Zhao, Y.Y., Mao, M.W., Zhang, W.J., Wang, J., Li, H.T., Yang, Y., Wang, Z. and Wu, J.W. (2018) Expanding RNA binding specificity and affinity of engineered PUF domains. Nucleic Acids Res, 46, 4771-4782.

36. Jarmoskaite, I., Denny, S.K., Vaidyanathan, P.P., Becker, W.R., Andreasson, J.O.L., Layton, C.J., Kappel, K., Shivashankar, V., Sreenivasan, R., Das, R. et al. (2019) A Quantitative and Predictive Model for RNA Binding by Human Pumilio Proteins. Mol Cell, 74, 966-981 e918.

37. Wang, Y., Havel, J. and Beal, P.A. (2015) A Phenotypic Screen for Functional Mutants of Human Adenosine Deaminase Acting on RNA 1. ACS Chem Biol, 10, 2512-2519.

38. Kuttan, A. and Bass, B.L. (2012) Mechanistic insights into editing-site specificity of ADARs. Proc Natl Acad Sci U S A, 109, E3295-3304. 
602

603

604

605

606

607

608

609

610

611

612

613

614

615

616

617

618

619

620

621

622

623

624

625

626

627

628

629

630

631

632

633

634

635

636

637

638

639

640
39. Li, H. and Durbin, R. (2009) Fast and accurate short read alignment with BurrowsWheeler transform. Bioinformatics, 25, 1754-1760.

40. Li, H., Handsaker, B., Wysoker, A., Fennell, T., Ruan, J., Homer, N., Marth, G., Abecasis, G., Durbin, R. and Genome Project Data Processing, S. (2009) The Sequence Alignment/Map format and SAMtools. Bioinformatics, 25, 2078-2079.

41. Picardi, E., D'Erchia, A.M., Montalvo, A. and Pesole, G. (2015) Using REDItools to Detect RNA Editing Events in NGS Datasets. Curr Protoc Bioinformatics, 49, 1212 11-12 1215.

42. Dobin, A., Davis, C.A., Schlesinger, F., Drenkow, J., Zaleski, C., Jha, S., Batut, P., Chaisson, M. and Gingeras, T.R. (2013) STAR: ultrafast universal RNA-seq aligner. Bioinformatics, 29, 15-21.

43. Wagih, O. (2017) ggseqlogo: a versatile $R$ package for drawing sequence logos. Bioinformatics, 33, 3645-3647.

44. Lehmann, K.A. and Bass, B.L. (2000) Double-stranded RNA adenosine deaminases ADAR1 and ADAR2 have overlapping specificities. Biochemistry, 39, 12875-12884.

45. Bohn, M.F., Shandilya, S.M.D., Silvas, T.V., Nalivaika, E.A., Kouno, T., Kelch, B.A., Ryder, S.P., Kurt-Yilmaz, N., Somasundaran, M. and Schiffer, C.A. (2015) The ssDNA Mutator APOBEC3A Is Regulated by Cooperative Dimerization. Structure, 23, 903-911.

46. Prasad, K.M., Xu, Y., Yang, Z., Acton, S.T. and French, B.A. (2011) Robust cardiomyocyte-specific gene expression following systemic injection of AAV: in vivo gene delivery follows a Poisson distribution. Gene Ther, 18, 43-52.

47. Zincarelli, C., Soltys, S., Rengo, G. and Rabinowitz, J.E. (2008) Analysis of AAV serotypes 1-9 mediated gene expression and tropism in mice after systemic injection. Mol Ther, 16, 1073-1080.

48. Gehrke, J.M., Cervantes, O., Clement, M.K., Wu, Y., Zeng, J., Bauer, D.E., Pinello, L. and Joung, J.K. (2018) An APOBEC3A-Cas9 base editor with minimized bystander and off-target activities. Nat Biotechnol, 36, 977-982.

49. Diroma, M.A., Ciaccia, L., Pesole, G. and Picardi, E. (2019) Elucidating the editome: bioinformatics approaches for RNA editing detection. Brief Bioinform, 20, 436-447.

50. Bahn, J.H., Lee, J.H., Li, G., Greer, C., Peng, G. and Xiao, X. (2012) Accurate identification of A-to-I RNA editing in human by transcriptome sequencing. Genome Res, 22, 142-150.

51. Bazak, L., Haviv, A., Barak, M., Jacob-Hirsch, J., Deng, P., Zhang, R., Isaacs, F.J., Rechavi, G., Li, J.B., Eisenberg, E. et al. (2014) A-to-I RNA editing occurs at over a hundred million genomic sites, located in a majority of human genes. Genome Res, 24, 365-376. 
bioRxiv preprint doi: https://doi.org/10.1101/2021.08.31.458316; this version posted August 31, 2021. The copyright holder for this preprint (which was not certified by peer review) is the author/funder, who has granted bioRxiv a license to display the preprint in perpetuity. It is made available under aCC-BY-NC-ND 4.0 International license.

TABLE AND FIGURES LEGENDS
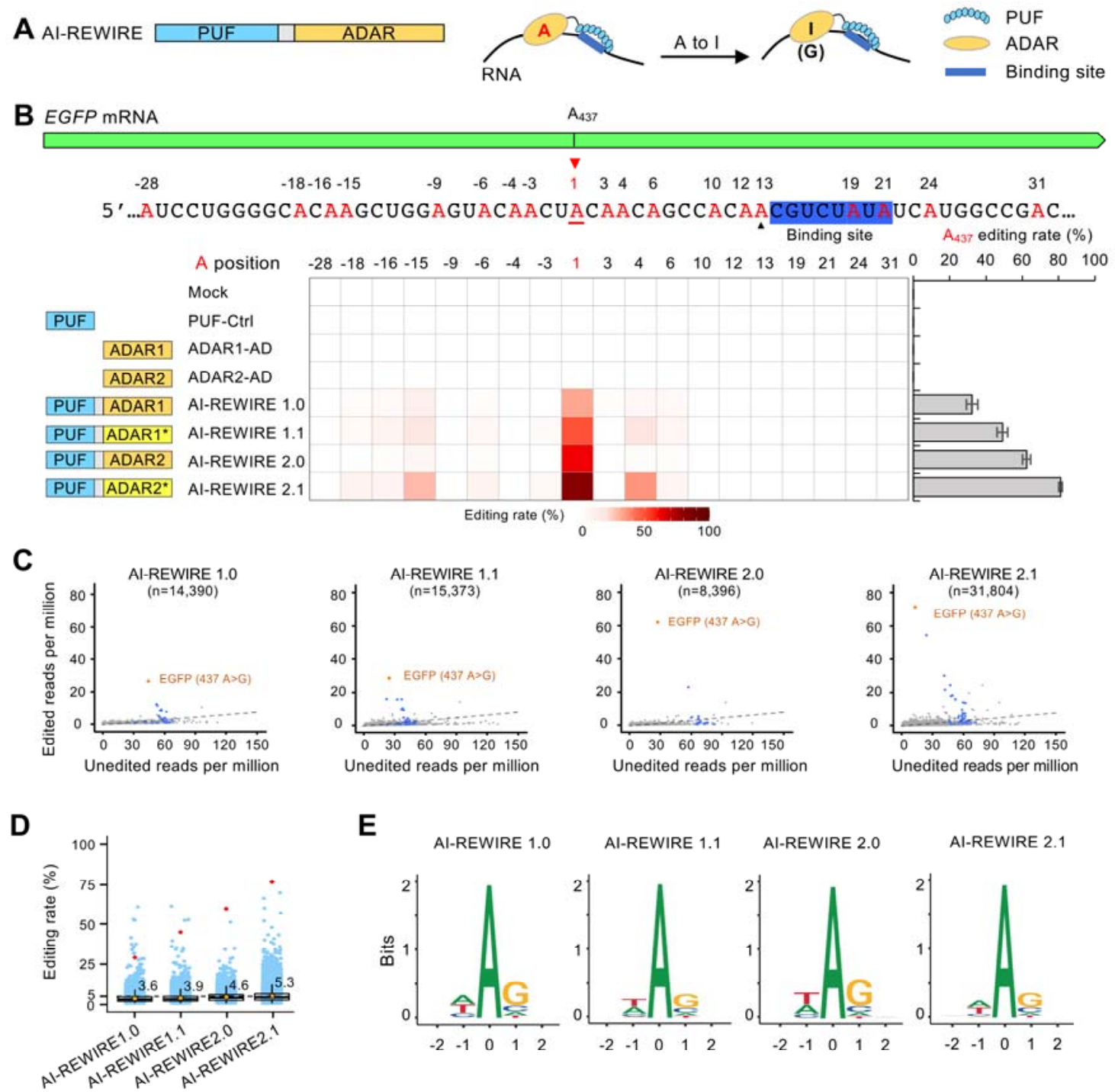

E

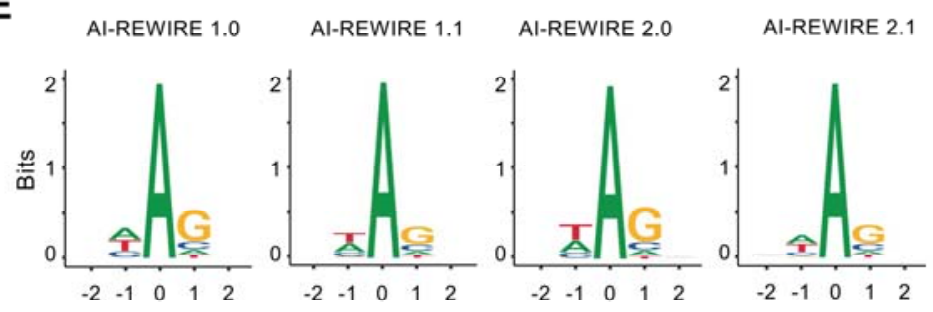

$\mathbf{F}$

GRIA2 mRNA

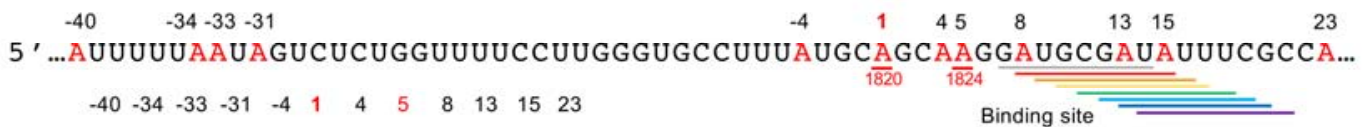
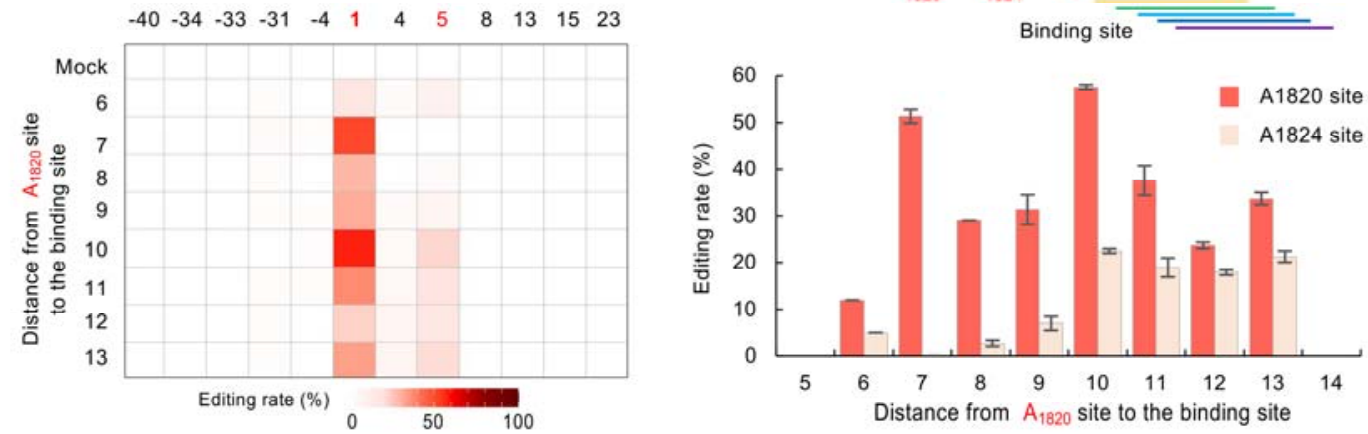

642 Figure 1. Engineering Al-REWIREs for A-to-I transformation at specific positions.

643 (A) Engineering AI-REWIREs for specifically A-to-I editing. Domain configuration of AI-REWIRE (left);

644 schematic of AI-REWIRE mediated specific A-to-I base editing on RNAs (right). 
645 (B) Efficient editing of EGFP mRNA by different versions of Al-REWIREs. The PUF binding site in 646 EGFP is highlighted in blue, with the nearby adenosines marked in red (top). Heatmap showed the 647 editing rates of all adenosines near the on-target site A437 (position 1) for each AI-REWIRE and 648 control. The on-target editing rate of A437 was shown at right. The editing rates presented were 649 measured by RNA-seq with triplicates (see methods).

650 (C) Scatter plots of transcriptome-wide A-to-I RNA editing in the samples treated with Al-REWIREs. 651 For each edited adenosine, the numbers of unedited reads ( $x$-axis) and edited reads ( $y$-axis) were 652 plotted. The on-target site A437 is highlighted in orange, and the adenosines near the targeted site (in 653 a 400-nt window) are labelled in blue. $n$, numbers of all edited adenosines detected. The $5 \%$ editing 654 rate was indicated with dashed lines. Three independent experiments were performed (see methods).

655 (D) Editing rates of transcriptome-wide A-to-I RNA editing in the samples treated with Al-REWIREs 656 (data from Figure 1C with minimum coverage cutoff at 100). On-target sites are represented by red 657 rhombuses; average editing rates of off-targets in each sample are indicated with orange dots and 658 values are nearby; the $5 \%$ editing rate was indicated with a dashed line.

659 (E) Sequence logos derived from edited adenosines. The analyses were conducted using RNA-seq 660 data from samples treated with different AI-REWIREs (data from Figure 1C).

661 (F) Editing of exogenously expressed GRIA2 mRNA by Al-REWIRE1.0 that binds at different 662 distances from the intended editing site (A1820). Left, the editing rates of all adenosines near the 663 binding site were plotted against the distance from the editing site to the 5' end of PUF binding site. 664 Right, the editing rates of two major sites were plotted against the binding distance (mean \pm s.e.m. $665 \mathrm{n}=3)$. 
A

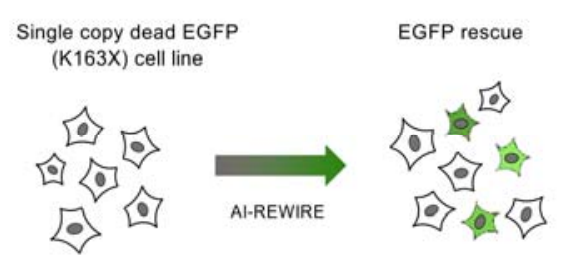

B

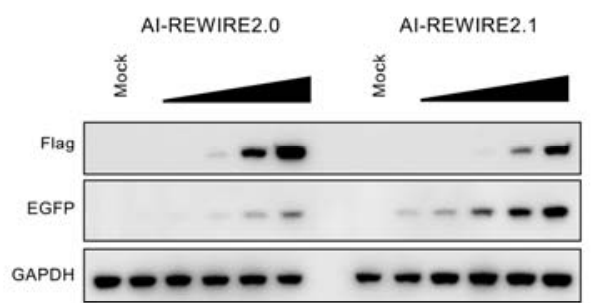

C

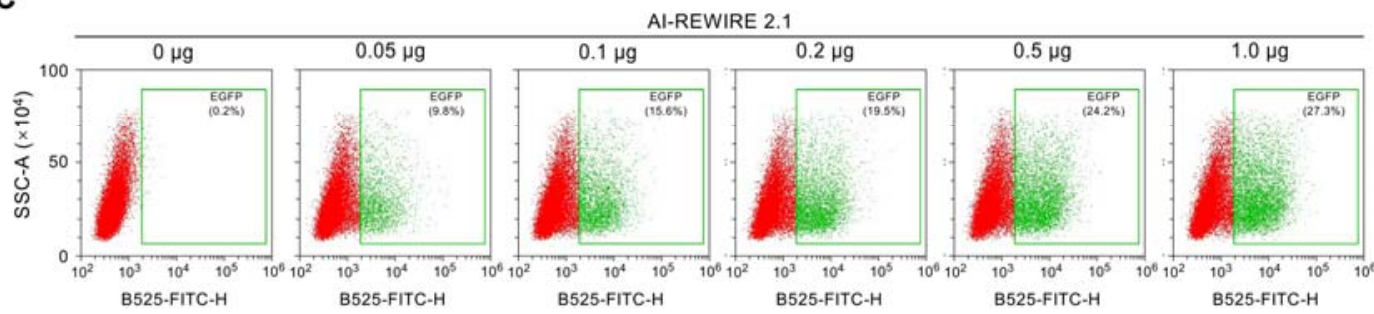

D

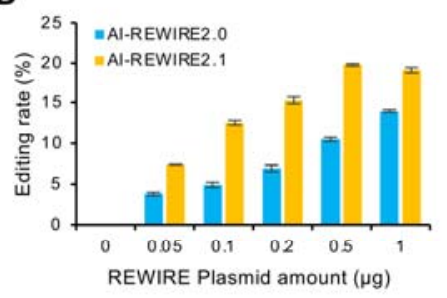

E

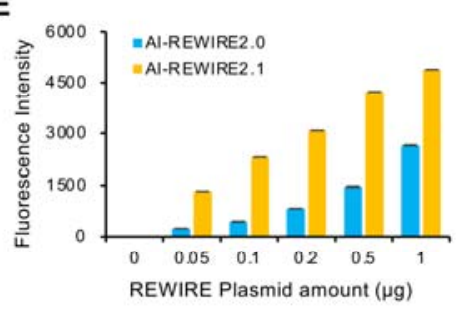

$\mathbf{F}$

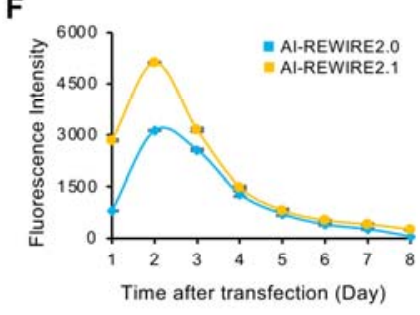

G
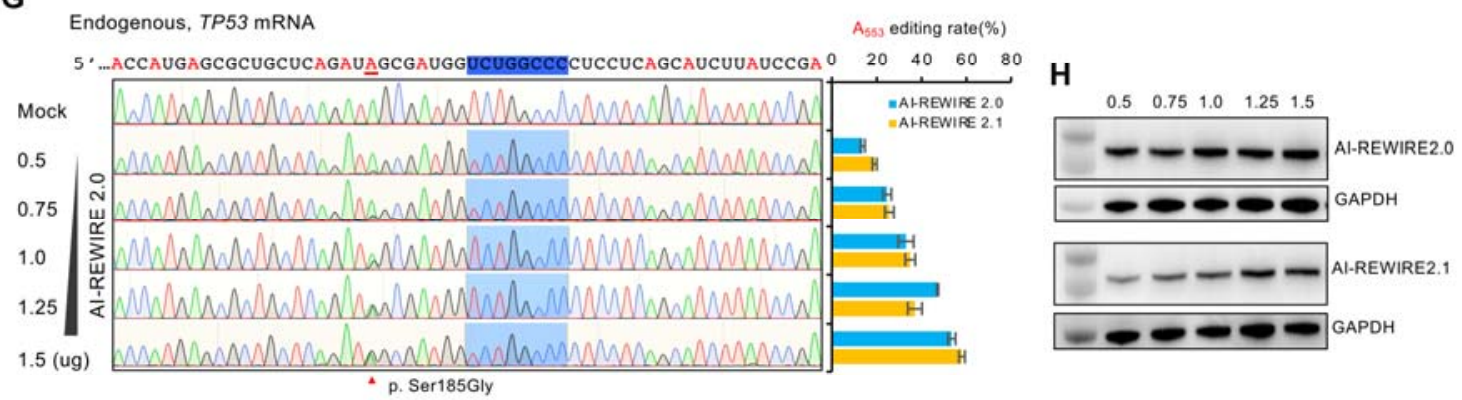

I

Exogenous, DMD: c.1682G>A (p.Trp561Ter) mRNA

$\begin{array}{llllll}-18-17-15-13 & -4-3-2 & 1 & 4 & 5 & 12\end{array} 16171920$

... C A A G A C A U C CUUCUCA A A AGC A ACGUCOU CUG G A C...
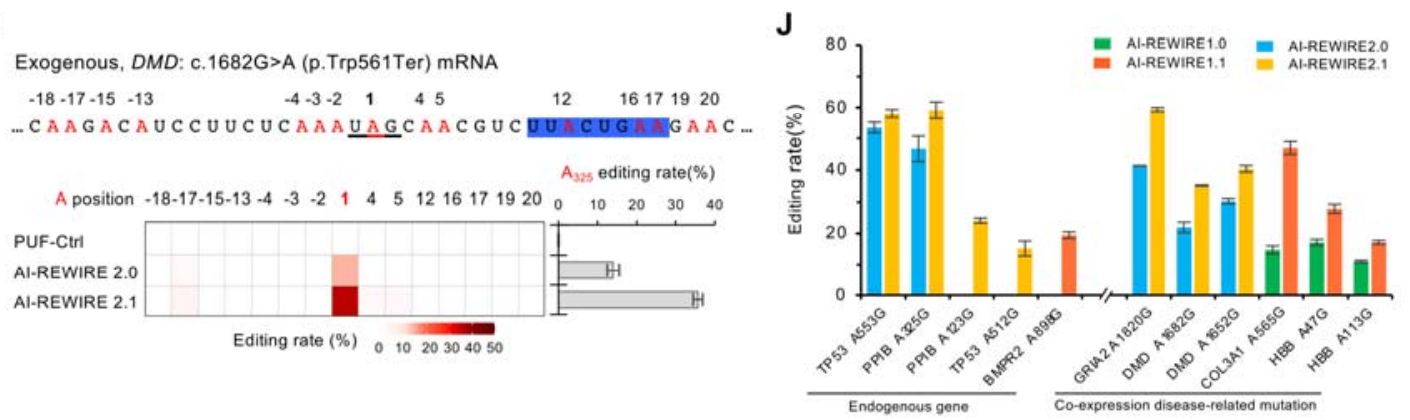

667

668

Figure 2. Application of AI-REWIREs.

669 (A) Using Al-REWIREs to rescue dead EGFP. The K163X mutation was introduced to create a 670 premature stop codon in the EGFP gene that was stably integrated into 293 cells with Flp-In system.

671 The A-to-I editing on this site can restore the translation of full-length EGFP.

672 (B) Western blot of expression level of Al-REWIREs and the rescued EGFP, with detection of GAPDH 673 as loading controls. 
674 (C) Flow cytometry of dead EGFP cell lines transfected with different amounts of AI-REWIRE2.1 using

675 a 12-well plate. The cells were analyzed 48h post transfection.

676 (D) Editing rates of the K163X (measured by Sanger sequencing) in dead EGFP cells. The 677 experiment conditions were same as panel $\mathrm{C}$.

678 (E) Fluorescence intensity of the rescued dead-EGFP cells. The experiment conditions were same as 679 panel C.

680 (F) Duration of RNA editing by the AI-REWIRE2.0/2.1, judged by the fluorescence intensity at each 681 day after a single transfection of $1 \mu$ g plasmid.

682 (G) Al-REWIREs edited the TP53 A553 site in a dose-dependent manner (see methods). The editing 683 rates of all adenosines nearby were measured by Sanger sequencing (Left), with the editing rate of 684 the on-target $A 553$ site shown in right. Values represent mean \pm s.e.m. $n=3$.

685 (H) Western blot of expression level of AI-REWIREs in different co-expression experiments (samples 686 are consistent with Figure 2G). The Al-REWIERs were Flag-tagged and the GAPDH were used as a 687 loading control. The western blot was done in technical duplicate.

688 (I) A-to-I editing of DMD $1682 \mathrm{G}>\mathrm{A}$ mutation that is associated with Duchenne muscular dystrophy 689 disease, the Deep-seq data showing the $1682 \mathrm{G}>\mathrm{A}$ in DMD is corrected to varying levels with different 690 Al-REWIREs.

691 (J) Editing of exogenous disease-related $\mathrm{G}>\mathrm{A}$ mutations and endogenous sites using Al-REWIREs 692 specifically designed to recognize the target mRNAs. The mutations were selected from ClinVar. 693 (mean \pm s.e.m. $n=3$ ).

694 
A CU-REWIRE

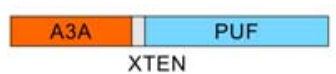

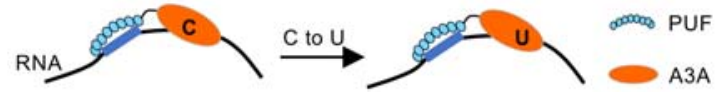

EGFP mRNA

C504

B

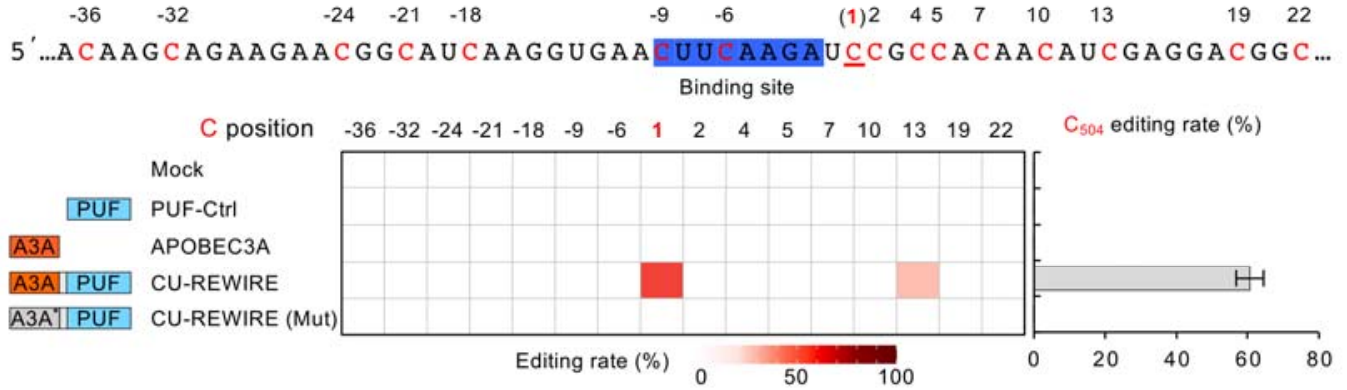

C

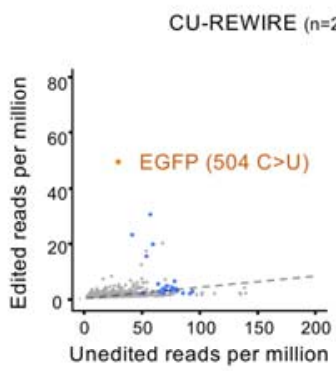

E

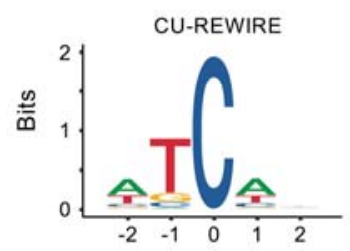

D
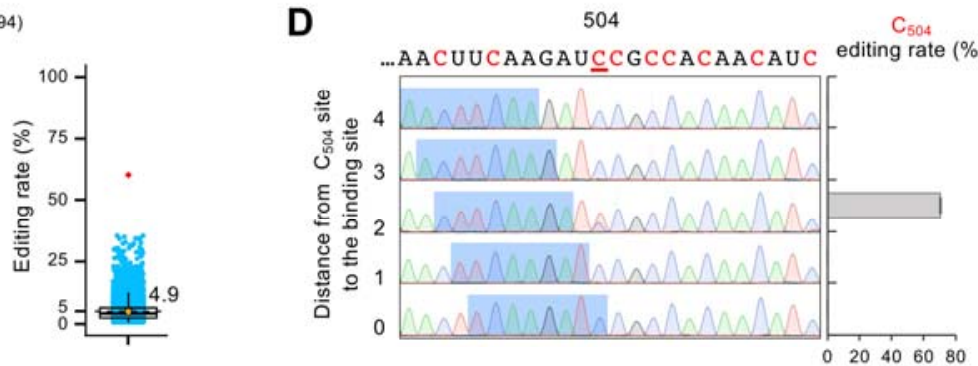

$\mathbf{F}$

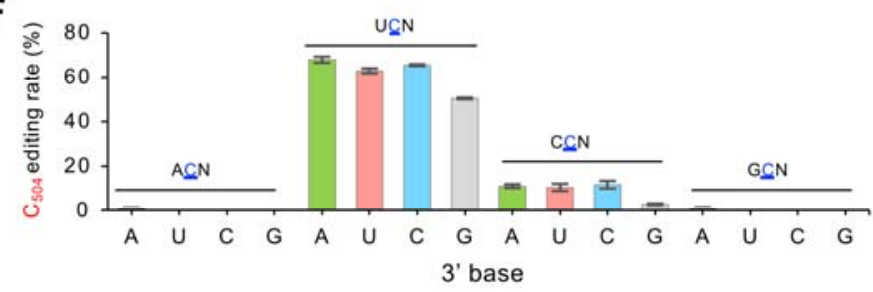

G Endogenous, CTNNB1 mRNA

H
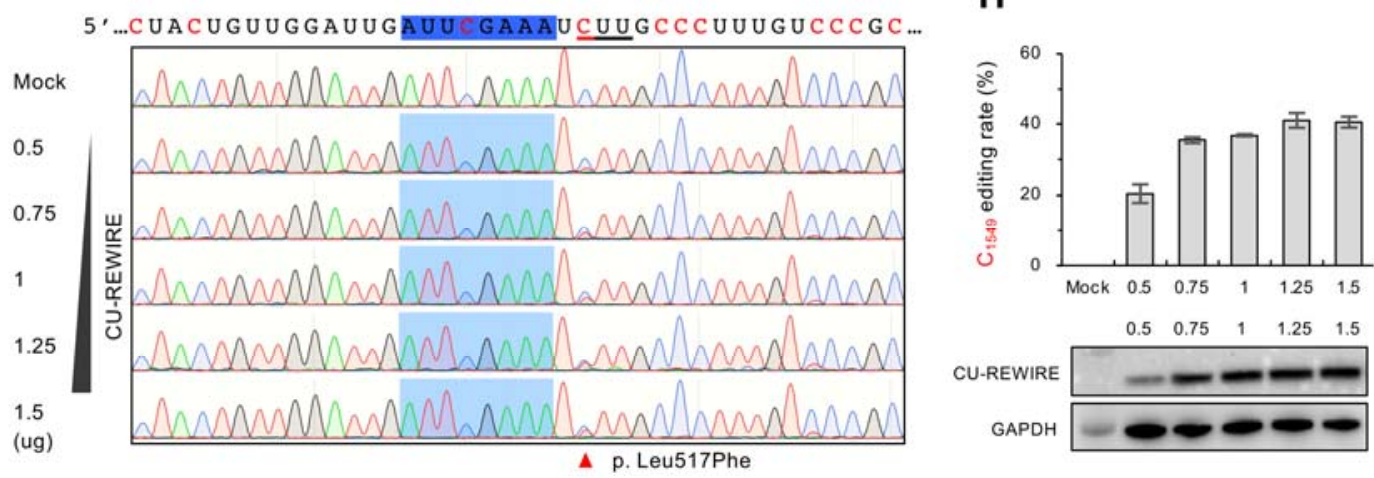

696

697 Figure 3. Engineering CU-REWIREs for targeted C-to-U conversion.

698 (A) Engineering CU-REWIRE for specific C-to-U editing. Left, Domain configuration of CU-REWIRE;

699 right, schematic of CU-REWIRE mediated specific C-to-U base editing on RNAs. 
700 (B) Efficient editing of EGFP mRNA by CU-REWIRE. The PUF binding site in EGFP and the nearby

701 cytidines were marked (The experiments and analyses are similar to Figure 1B), with the C504 as the

702 on-target editing site. CU-REWIRE with mutated deaminase domain was included as negative control.

703 (C) Transcriptome-wide C-to-U RNA editing in the sample treated with CU-REWIRE (labels and cutoff

704 are similar with Figure 1C and 1D). Left: the on-target site C504 is highlighted in orange, and the

705 cytidines near the targeted site are labelled in blue; right: the on-target site C504 is highlighted in red;

$706 \mathrm{n}$, numbers of all edited cytidines detected. The $5 \%$ editing rate was indicated with dashed lines.

707 (D) On-target editing rates of CU-REWIRE with different editing distances from binding sites, which

708 represent the number of bases between the editing site and 3' end of the PUF binding site.

709 (E) Sequence logos derived from edited cytidines. The analyses were conducted using RNA-seq data

710 from samples treated with different CU-REWIRE (data from Figure 3C).

711 (F) Editing activity of CU-REWIRE on all combinations of 3-nt flanking bases around the candidate 712 cytidine on the EGFP transcript.

713 (G) Editing of CTNNB1 mRNA with different doses of CU-REWIREs expression vectors. The editing 714 rates of all cytidines nearby the PUF binding site were measured by Sanger sequencing. Values 715 represent mean \pm s.e.m. $n=3$.

716 (H) Editing rates of on-target C1549 at CTNNB1 with different amounts of CU-REWIREs. The rates 717 were measured by Sanger sequencing (top). Values represent mean \pm s.e.m. $n=3$. The protein 718 expression level of CU-REWIREs in different co-expression experiments with GAPDH as a loading 719 control (bottom). The western blot was done in technical duplicate. 
bioRxiv preprint doi: https://doi.org/10.1101/2021.08.31.458316; this version posted August 31, 2021. The copyright holder for this preprint (which was not certified by peer review) is the author/funder, who has granted bioRxiv a license to display the preprint in perpetuity. It is made available under aCC-BY-NC-ND 4.0 International license.

A

Original Al-REWIRE RNA 5 ;...

Upgraded AI-REWIRE

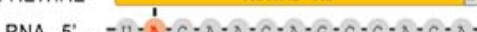

B

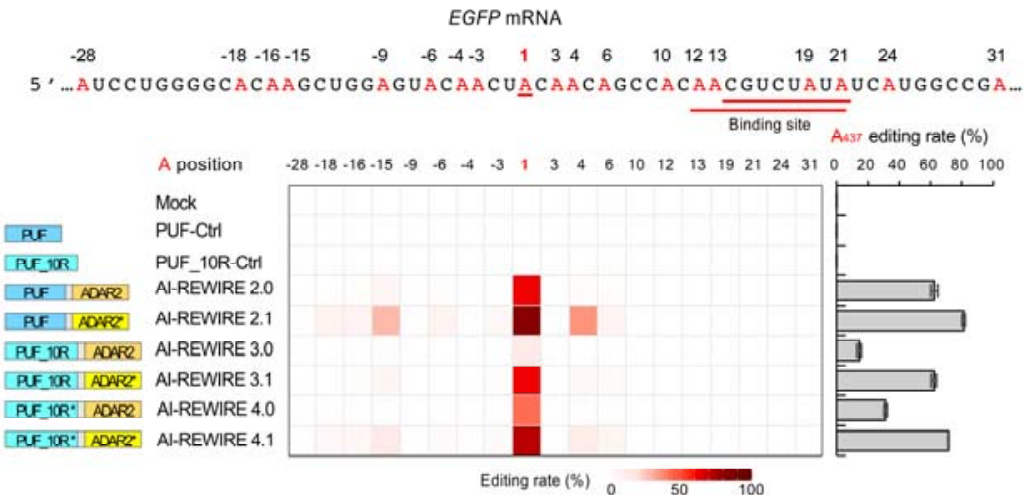

D

Original CU-REWIRE

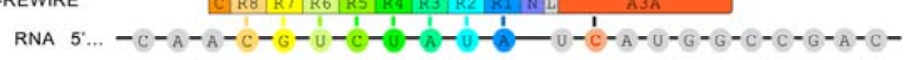

\begin{tabular}{ll|l|l|l|l|l|l|l|l|l|l|} 
Upgraded CU-REWIRE $\quad$ C R10 R9 & R8 & R7 & R6 & R5 & R4 & R3 & R2 & RI & N L & A3A \\
\hline
\end{tabular}

RNA 5. $-C-A-A-C-G-U-C-B-B-B-B-0-C-A-U-G-G-C-C-G-A-C-$

$E$

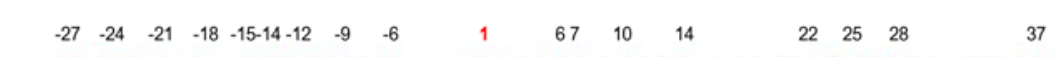

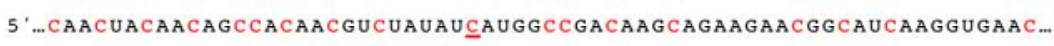
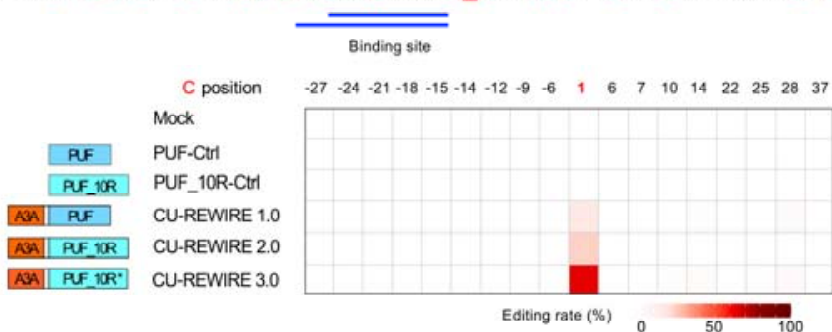

Cass editing rate $(\%)$
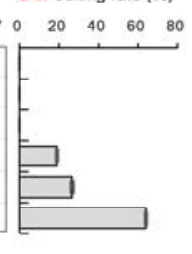

C
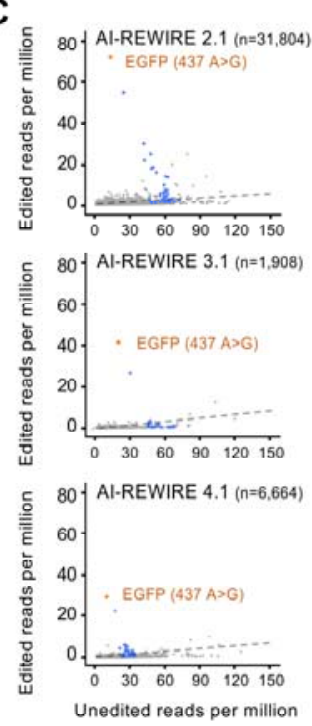

$\mathbf{F}$
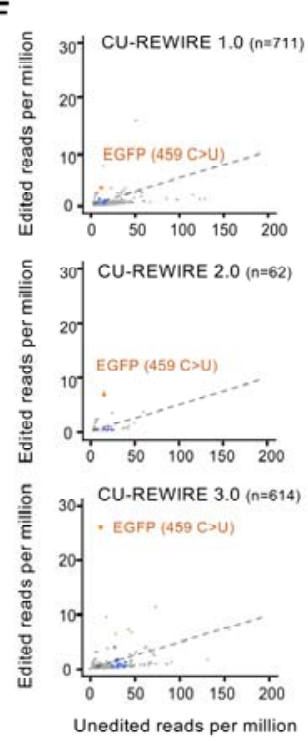

G

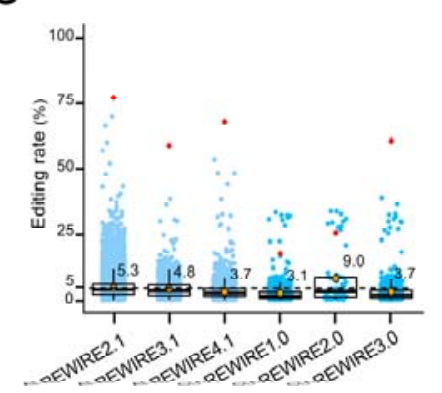

H A

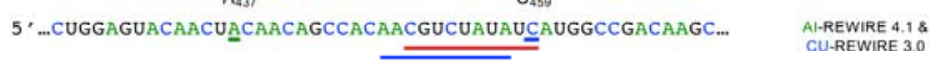
Binding site
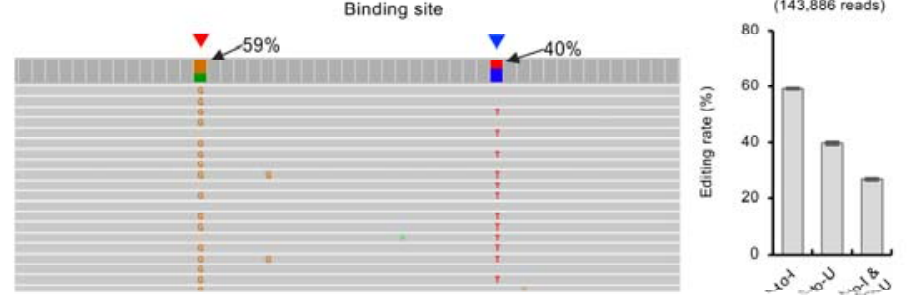

724 (A) Schematic of Al-REWIREs with upgraded PUF variants. Top, the original AI-REWIRE with the 725 PUF-8R that recognize targets by 8-nt sequence. Bottom, upgraded AI-REWIRE with the PUF-10R 726 that recognize targets by 10 -nt sequence.

727 (B) Efficient editing of EGFP mRNA by upgraded versions of Al-REWIRE. The PUF binding site in 728 EGFP is underlined in red. The experiments and analyses were similar to Figure 1B. The AI-REWIRE 
729 3.0, 3.1, 4.0 and 4.1 all used PUF_10R scaffold as target recognition domain, with 4.0 and 4.1

730 containing a short loop between PUF repeat 9 and 10 (see method).

731 (C) Scatter plots of transcriptome-wide A-to-I RNA editing with edited/unedited reads (labelled as

732 Figure 1C) for the samples treated with AI-REWIREs.

733 (D) Schematic of CU-REWIREs with upgraded PUF variants.

734 (E) Efficient editing of EGFP mRNA by upgraded versions of CU-REWIRE. The PUF binding site in

735 EGFP is underlined in blue, with the nearby cytosines marked in red (on-target site, C459). The

736 experiments and analyses were similar to Figure 3B. The CU-REWIRE 2.0 and 3.0 all use PUF_10R

737 as target recognition domain (see methods).

738 (F) Scatter plots of transcriptome-wide C-to-U RNA editing in the samples treated with CU-REWIREs

739 (labeled as Figure 3C left panel).

740 (G) Editing rates of transcriptome-wide RNA editing in samples treated with different REWIREs (data

741 from Figure 4C and 4F, labels and cutoff are same as Figure 1D).

742 (H) Successive editing of adenosine and cytidine with the Al-REWIRE 4.1 and CU-REWIRE 3.0 that

743 bind to the same site on EGFP (the binding site is underlined in red and blue respectively). The

744 experimental condition is similar with Figure 4B and 4E except that half amounts of each vector were

745 transfected. The experiments and sequencing were carried out in duplicates. Left, representative

746 Deep-seq reads surrounding the target site; right, the editing rates calculated using the number of

747 reads supporting single and concurrent editing divided by the total reads. 
A

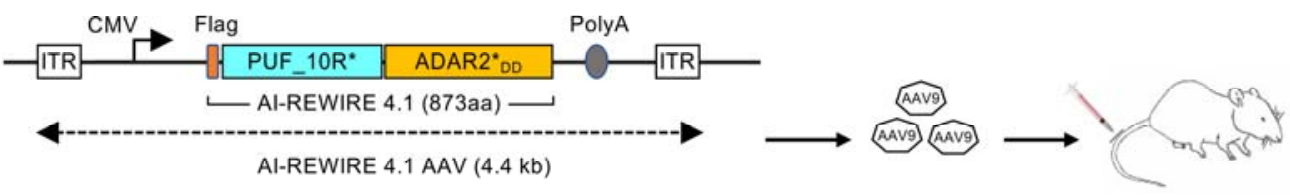

B EGFP MRNA

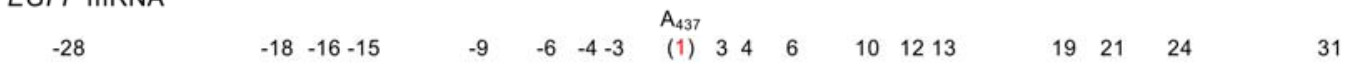

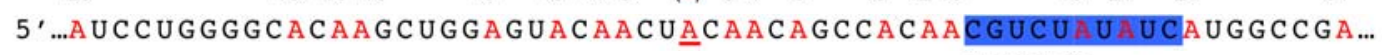
Binding site $\quad \mathrm{A}_{437}$ editing rate(\%)

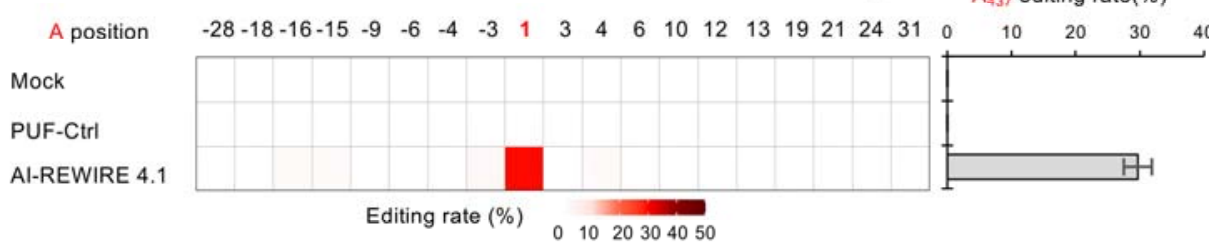

C

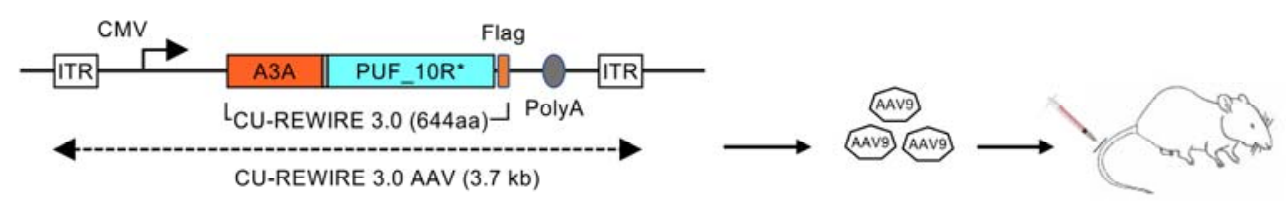

D EGFP mRNA

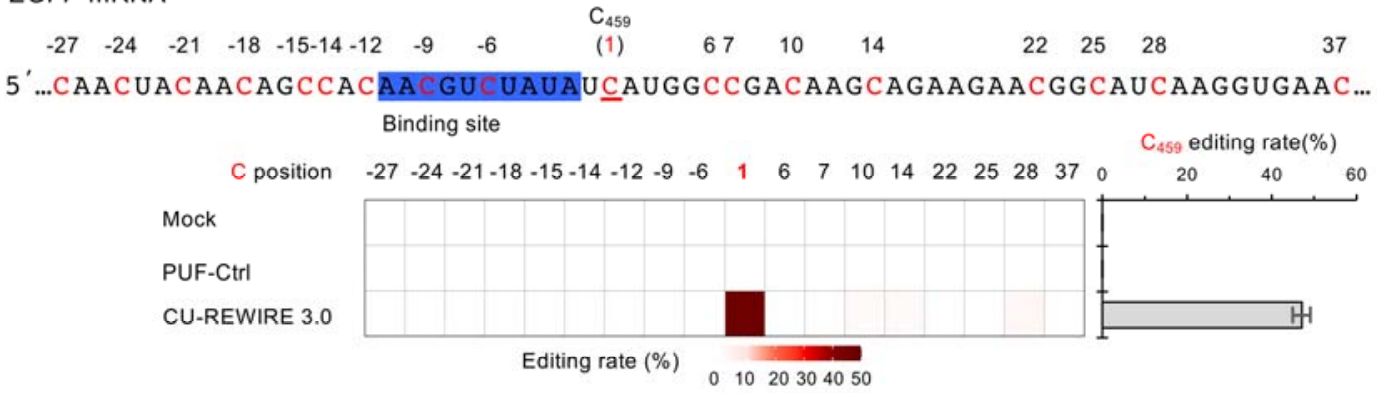

$\mathbf{E}$

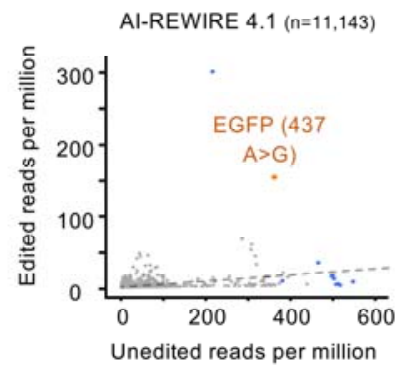

CU-REWIRE $3.0(n=9,260)$

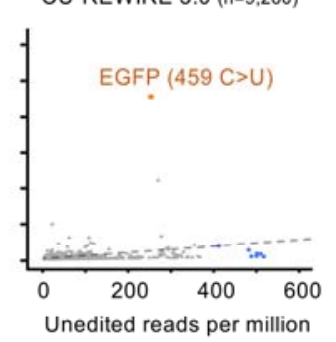

$\mathbf{F}$

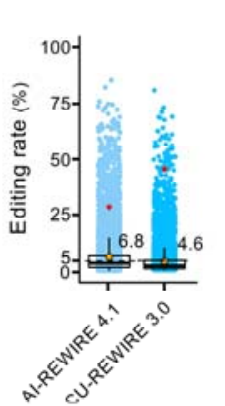

G

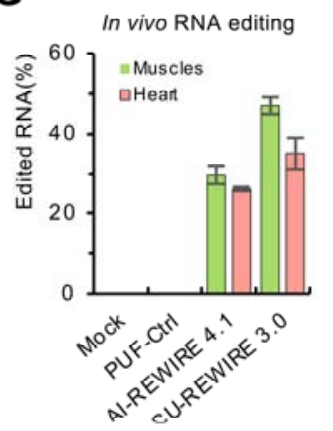

Figure 5. In vivo RNA editing in the B6-EGFP mouse model

750 (A) Schematic for AI-REWIRE 4.1 delivered to B6-EGFP mice through tail vein IV injection of AAV9.

751 The total insert size between the two inverted terminal repeats (ITR) was $4.4 \mathrm{~kb}$.

752 (B) Efficient Editing of EGFP mRNA by AI-REWIRE 4.1 in the tibialis anterior muscles. The PUF_10R

753 binding site and the on-target editing site A437 (position 1) were highlighted. The editing rates were 
754 measured by RNA-seq with triplicates as described in Figure 1B. Values represent mean \pm s.e.m. $\mathrm{n}=$ 7553.

756 (C) Schematic for CU-REWIRE 3.0 delivered to B6-EGFP mice using AAV9. The total insert size 757 between the two ITR was $3.7 \mathrm{~kb}$.

758 (D) Efficient editing of EGFP mRNA by CU-REWIRE 3.0 in the tibialis anterior muscles. The 759 experiments were similar to Figure 5B. Values represent mean \pm s.e.m. $\mathrm{n}=3$.

760 (E) Scatter plots of transcriptome-wide A-to-I or C-to-U RNA editing in the samples treated with 761 respective REWIREs (labels are similar with Figure $1 \mathrm{C}$ and Figure $3 \mathrm{C}$ left panel).

762 (F) Editing rates of transcriptome-wide RNA editing in samples treated with different REWIREs (data 763 from Figure 5E, labels and cutoff are same as Figure 1D and Figure 3C right panel).

764 (G) Efficient editing of EGFP mRNA in muscle and heart tissues. Values represent mean \pm s.e.m. $\mathrm{n}=$ 7653 , respectively. 


\section{Supplementary Figures.}

A
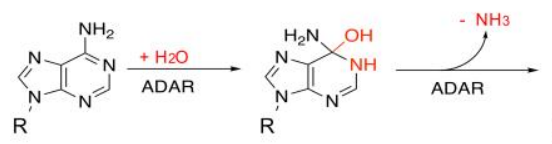

Adenosine (A)

Hydrated intermediate<smiles></smiles><smiles>[R]N1C=CC(N)(O)NC1=O</smiles>

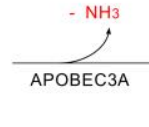

Cytidine $(\mathrm{C})$

Hydrated intermediate
B

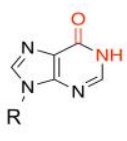

Inosine (I)

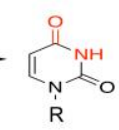

Uridine $(U)$

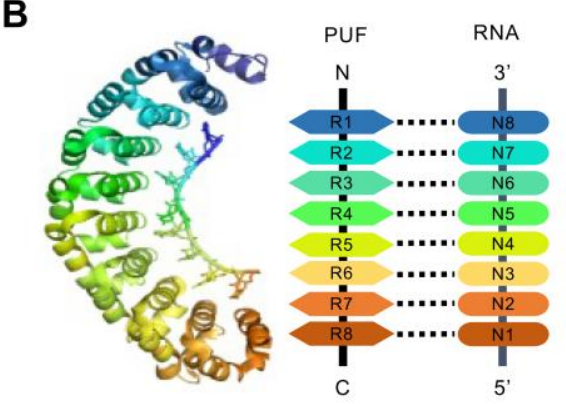

C

EGFP mRNA Edited RNA(\%)
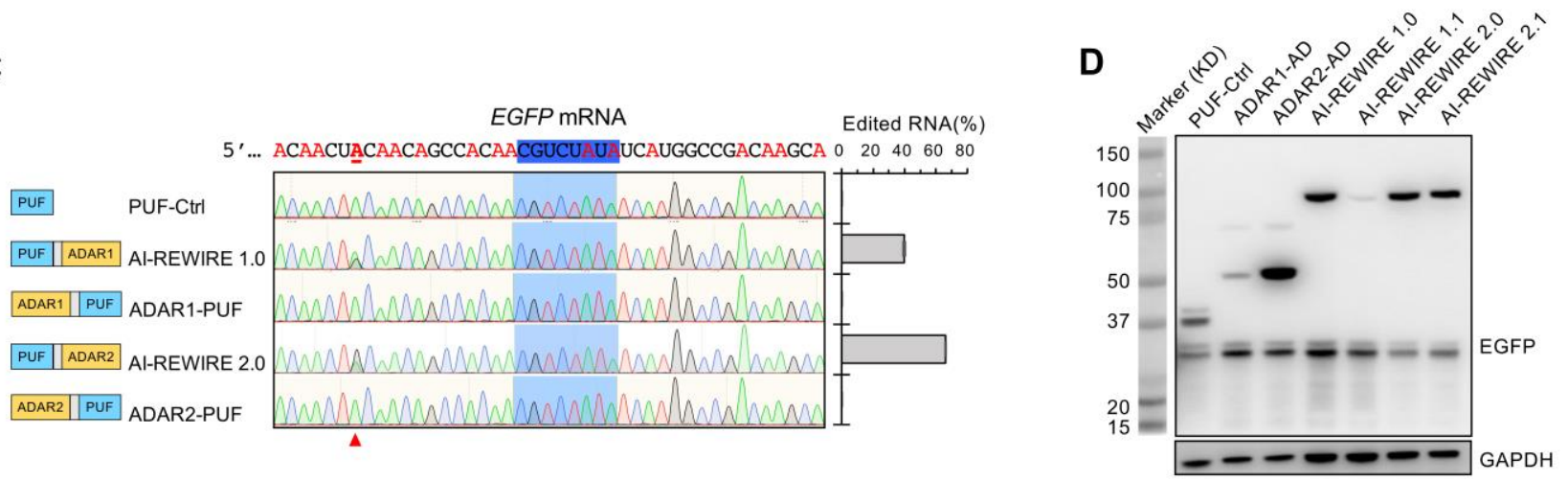

E

EGFP mRNA

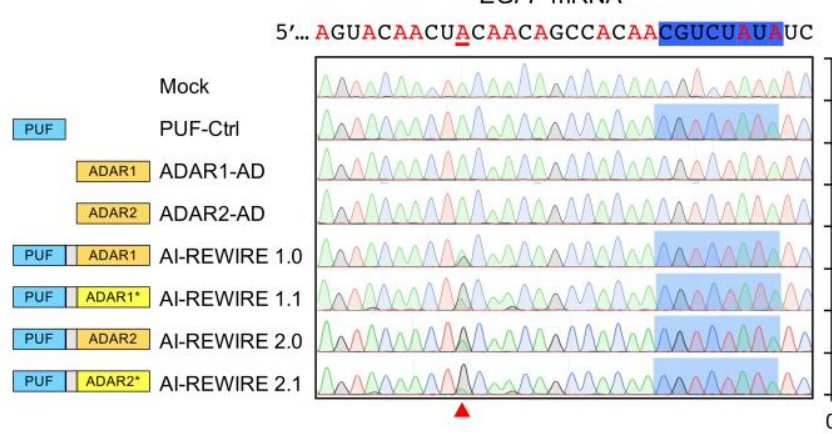

$\mathrm{A}_{437}$ editing rate(\%)

$\mathbf{F}$

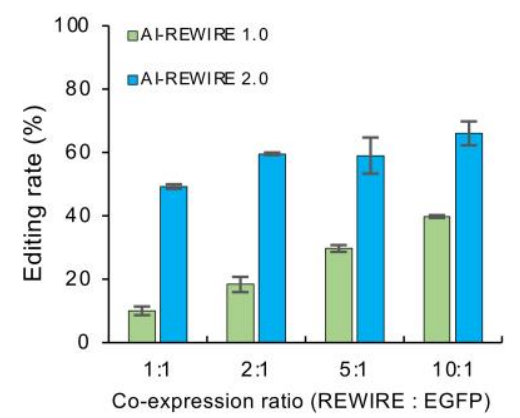

2

Figure S1. The design principle of REWIREs and assessment of specific A-to-I editing by AI-REWIREs.

(A) Top, the adenosine $(\mathrm{A})$ to inosine (I) editing reaction mediated by ADARs in vivo, where inosine will be recognized as guanosine $(G)$ by DNA polymerases. Bottom, the cytidine $(C)$ to uridine $(U)$ editing reaction mediated by APOBEC3A. $R$, ribose in RNA.

(B) Crystal structure of PUF domain (PDB: 2YJY) and the diagram of PUF-RNA interaction. The PUF domain can directly recognize its targeted RNA sequence without the co-folding and assembly steps into a gRNA-protein complex of CRISPR-Cas system. 
10 (C) Efficient editing of EGFP transcripts by various configuration combinations of PUF-ADAR and ADAR-PUF

11 fusion proteins. Base editing rates were measured by Sanger sequencing.

12 (D) Bar plots showing the on-target RNA base editing rates using different ratio of targets and Al-REWIREs (see

13 methods). Base editing rates were measured by Sanger sequencing. Values represent mean \pm s.e.m. $(n=3)$.

14 (E) Protein levels of Al-REWIREs. Representative images of western blot were from the same samples in Figure

15 1B, GAPDH was included as a loading control. The western blot was performed with technical duplicate.

16 (F) Efficient editing of EGFP transcripts by various Al-REWIRE versions. For each version, the editing rate of any

17 adenosine near the target site was measured by Sanger sequencing (left panel). The editing rate of the on-target

18 site A437 was shown in the right panel. Values represent mean \pm s.e.m. $(n=3)$. 
A

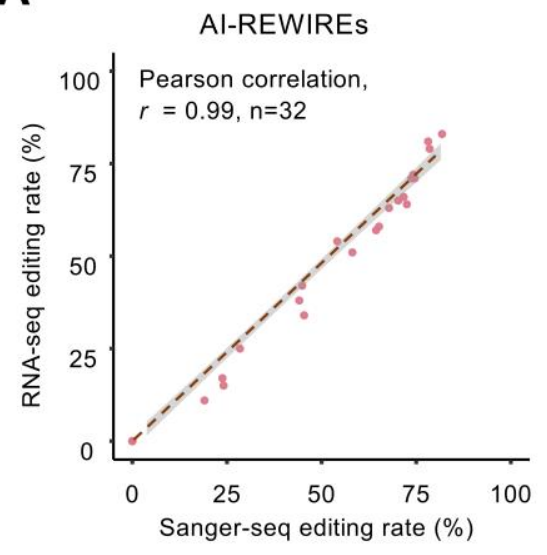

C

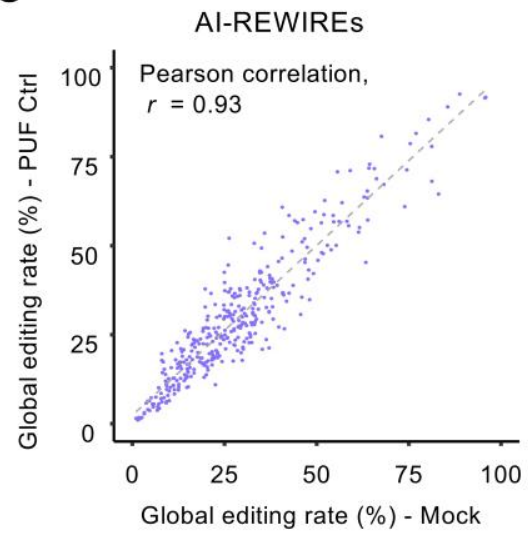

B

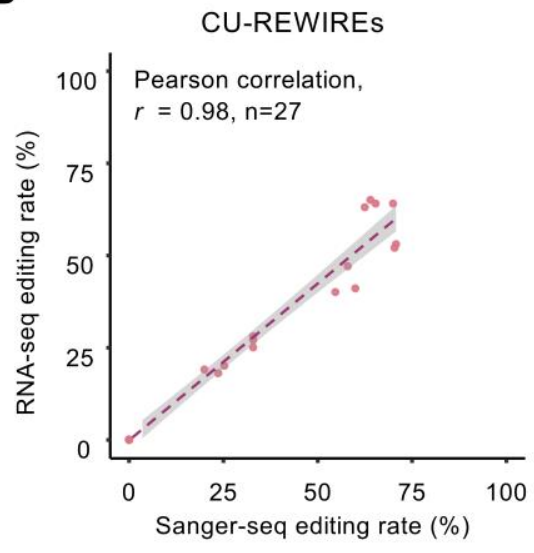

D

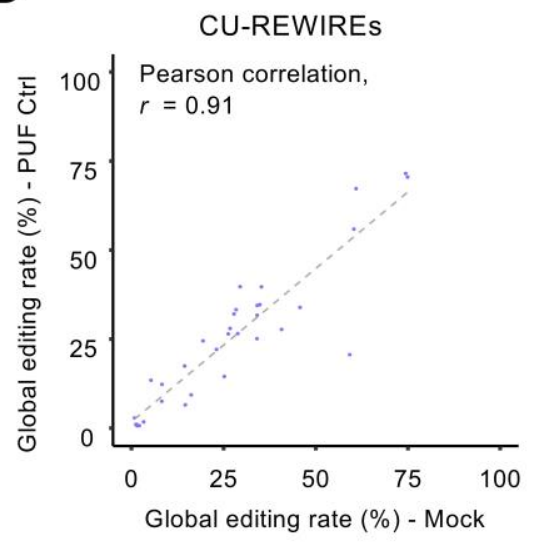

Figure S2. Sanger-seq and RNA-seq measurement correlation and transcriptome-wide off-target analysis of control RNA.

(A) Editing rate of Sanger-seq and RNA-seq measurement correlation on the target site edited by AI-REWIREs. Each dot, an individual A-to-I editing sample targeting EGFP mRNA used in this study; x-axis, on-target editing rate measured by Sanger-seq; y-axis, on-target editing rate measured by RNA-seq.

(B) Editing rate of Sanger-seq and RNA-seq measurement correlation on the target site edited by CU-REWIREs. Each dot, an individual C-to-U editing samples targeting EGFP mRNA used in this study; $\mathrm{x}$-axis, on-target editing rate measured by Sanger-seq; y-axis, on-target editing rate measured by RNA-seq.

(C) Transcriptome-wide off-target effects analysis of Mock control RNA and PUF-Ctrl RNA on native A-to-I editing sites. $x$-axis, editing rate in Mock sample; $y$-axis, editing rate in PUF-Ctrl sample. Three independent experiments were performed (see methods).

(D) Transcriptome-wide off-target effects analysis of Mock control RNA and PUF-Ctrl RNA on native C-to-U editing sites. $x$-axis, editing rate in Mock sample; y-axis, editing rate in PUF-Ctrl sample. Three independent experiments were performed. 
A

GRIA2 mRNA

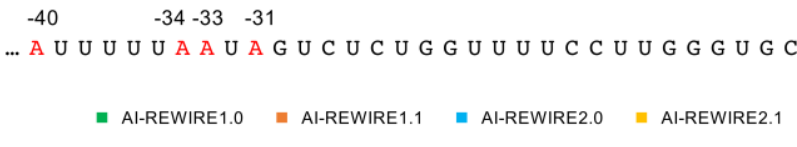
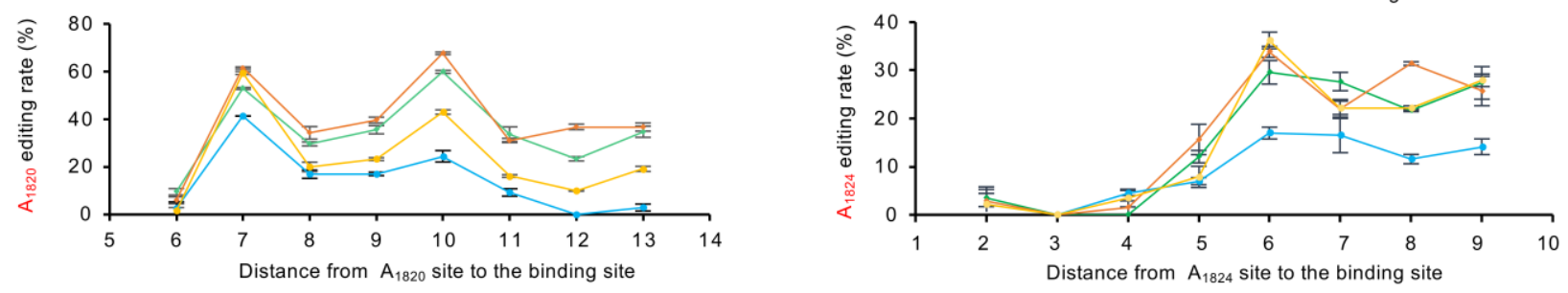

B

AI-REWIRE 1.0/1.1

C

AI-REWIRE 2.0/2.1
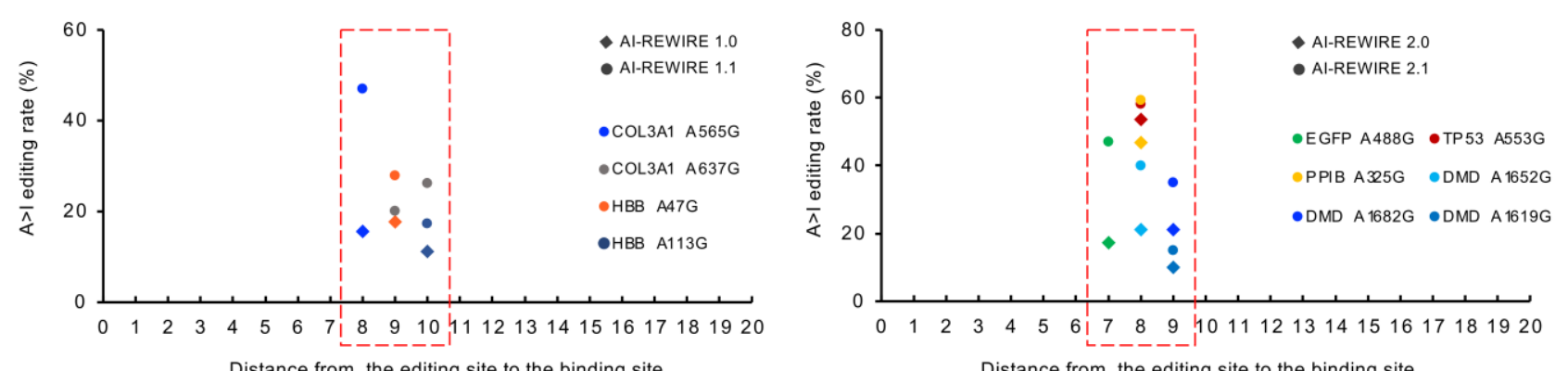

Distance from the editing site to the binding site

Distance from the editing site to the binding site

Figure S3. Al-REWIREs showed a narrow editing window.

(A) Designed editing on GRIA2 transcripts by AI-REWIREs with step-by-step strategy to test the optimal editing window. PUF binding sites in GRIA2 mRNA were underlined in different colors, with the nearby adenosines marked in red. Left, editing rates of GRIA2 A1820 site by different AI-REWIREs that binds with shifting distances from the A1820 site. Right, editing rates of GRIA2 A1824 site by the corresponding AI-REWIREs. For each version of AIREWIRE, the editing rate of adenosines near the targeted site were measured by Sanger sequencing. Values represent mean \pm s.e.m. $(n=3)$. from the editing site to the binding site, 1-based; $y$-axis, A-to-I editing rate. from the editing site to the binding site, 1-based; y-axis, A-to-I editing rate. 
A EGFPK163X mRNA
$-28$
$-21-19-18 \quad-15-13-1210-9$
$-4$
67
121315
$\begin{array}{lll}22 & 2425 & 27\end{array}$

5 '...AUG GCC GAC AAG CAG AAg AAC GGC AUC UAG GUG AAC UUC AAG IUC CGC CAC AAC AUC...

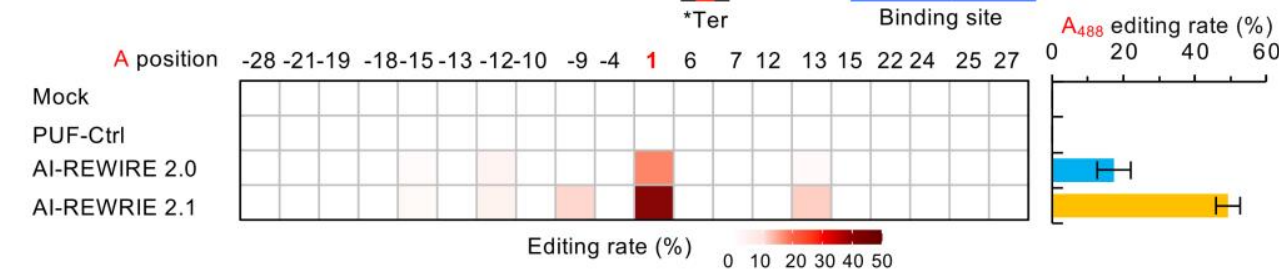

B

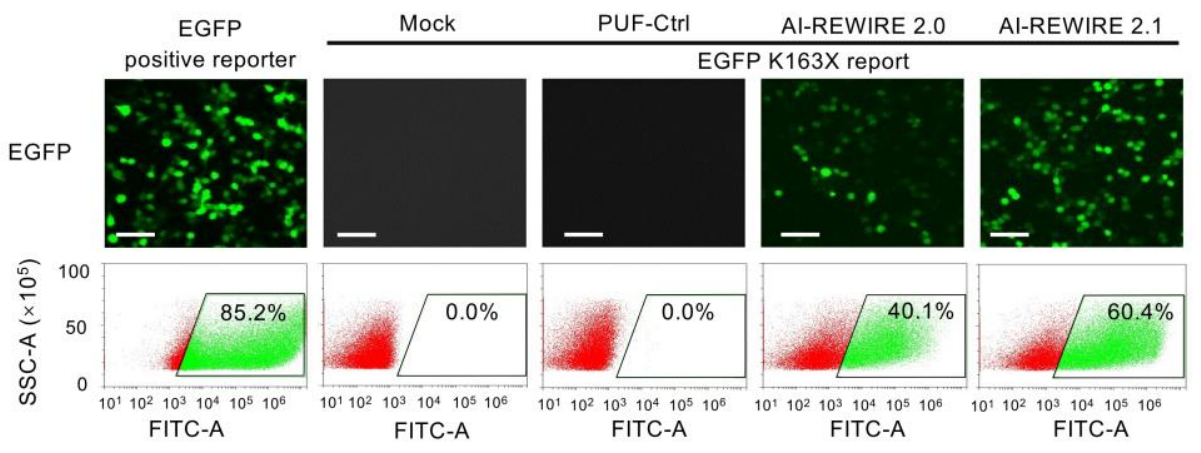

C Endogenous, PPIB mRNA

$5^{\prime} . .$. AUGAUCCAGGGCGAGACUUCACCAGGGGAGAUGGC C GGAGGAAaGAGCAUCUA $A_{325}$ editing rate(\%)

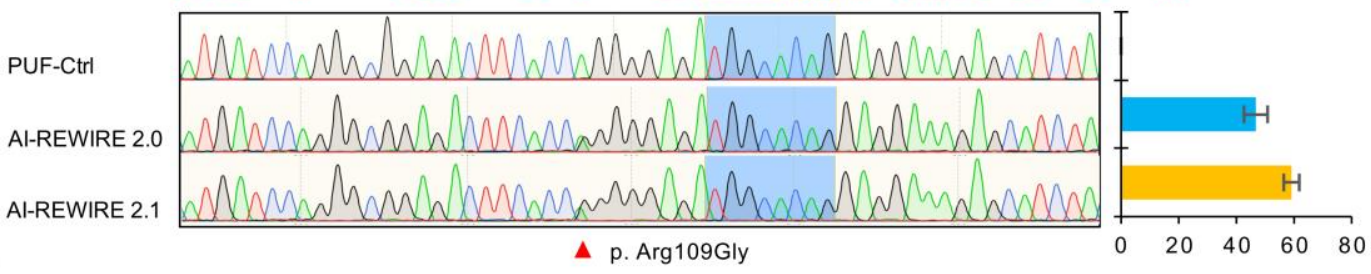

D Exogenous, GRIA2 mRNA

A p. Arg109Gly

$\mathrm{A}_{1820}$ editing rate(\%)

PUF-CtrI

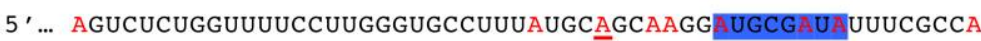

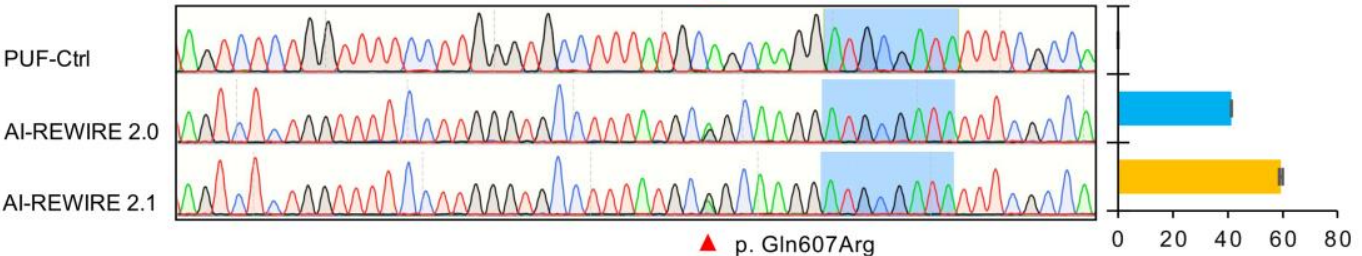

E

Exogenous, DMD: c.A1652G (p.Trp551Ter) mRNA

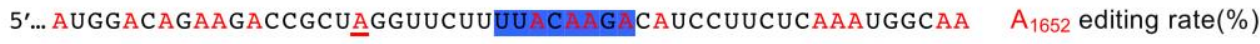

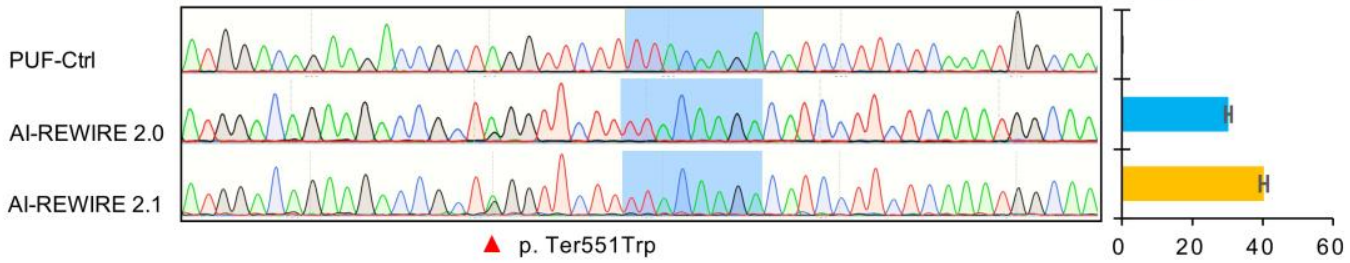

Figure S4. Application of AI-REWIREs on editing endogenous and exogenous pathogenetic mutant RNA. 
functional protein. The PUF binding site is highlighted in blue. Bottom, for each AI-REWIRE version, the editing rate of all adenosines near the target site was measured by Deep-seq (left) and the editing rate of the on-target site A437 was shown in right. Values represent mean \pm s.e.m. $(n=3)$.

(B) Restoration of EGFP fluorescence by the AI-REWIREs in same samples as judged by microscopy (top) and flow cytometry (bottom). Scale bar, $50 \mu$ m. Values represent mean \pm s.e.m. $(n=3)$.

(C) Editing rates for an endogenous gene, PPIB, with different AI-REWIREs. The editing rates of all adenosines nearby were measured by Sanger sequencing, with the editing rate of on-target A325 shown in right. Values represent mean \pm s.e.m. $(n=3)$.

(D) Engineering AI-REWIREs to edit GRIA2 A1820 site that is associated with ligand-gated ion channel in the central nervous system. The editing rates of all adenosines nearby were measured by Sanger sequencing (left), with the editing rate of on-target $A 1820$ shown on the right. Values represent mean \pm s.e.m. $(n=3)$. (E) Engineering Al-REWIREs to correct DMD 1652G>A mutation that is associated with Duchenne muscular dystrophy disease. The editing rates of all adenosines nearby were measured by Sanger sequencing (left), with the editing rate of on-target $\mathrm{A} 1652$ shown on the right. Values represent mean \pm s.e.m. $(n=3)$. 


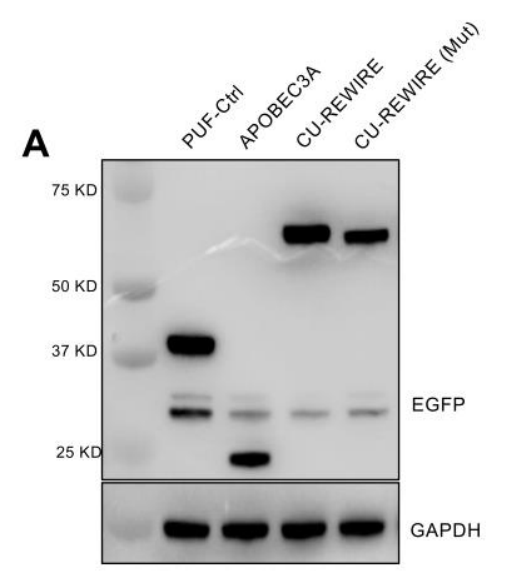

B Exogenous, EZH2: c.2191T>C (p.Tyr731His) mRNA

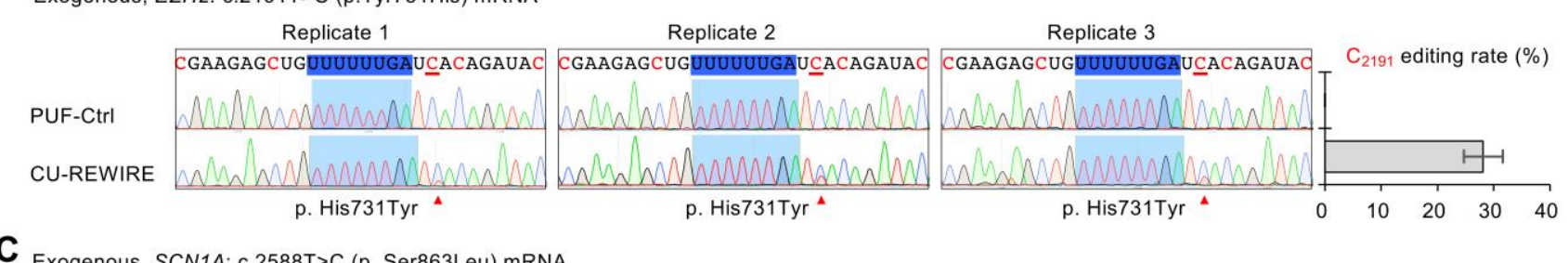

C Exogenous, SCN1A: c.2588T>C (p. Ser863Leu) mRNA

Replicate 1

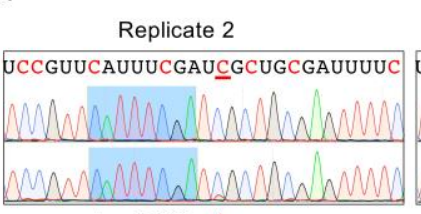

p. Leu863Ser ${ }^{\star}$
Replicate 3

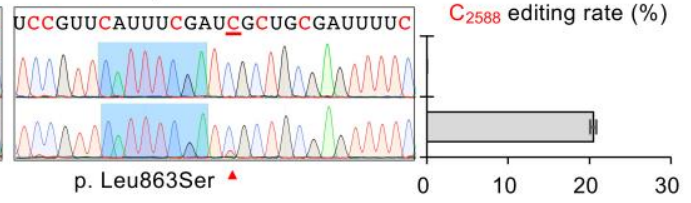

D

p. Leu863Ser

Replicate 2

Replicate 3

Replicate 1
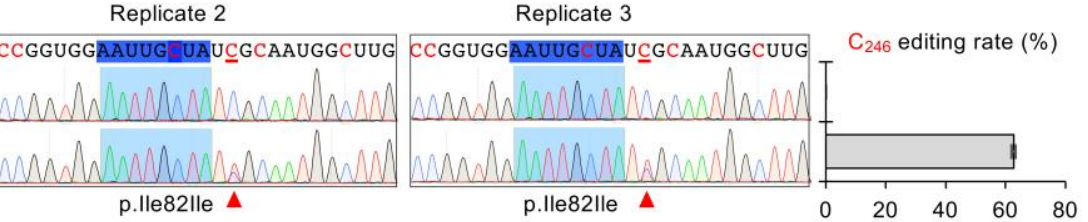

p.lle82lle $\Delta$

p.lle82lle 4

Figure S5. Protein expression level of CU-REWIREs and specific C-to-U base editing on exogenous pathogenetic mutant RNA.

(A) Protein expression level of PUF-control, APOBEC3A-control and CU-REWIREs. Representative images of western blot were from the same samples in Figure 3B. The western blot was done in technical duplicate.

(B) Example of using CU-REWIRE to correct disease-related mutation. The EZH2 2191T >C mutation is associated with Weaver syndrome disease, and we designed a CU-REWIRE to convert the 2191C back to U. The editing rates of all cytidines nearby the PUF binding site were measured by Sanger sequencing (Left), with the editing rate of the on-target of C2191 shown on the right. Values represent mean \pm s.e.m. $(n=3)$. disease. The editing rates of all cytidines nearby the PUF binding site were measured by Sanger sequencing (Left), with the editing rate of the on-target of $\mathrm{C} 2588$ shown on the right. Values represent mean \pm s.e.m. $(n=3)$. 
bioRxiv preprint doi: https://doi.org/10.1101/2021.08.31.458316; this version posted August 31, 2021. The copyright holder for this preprint (which was not certified by peer review) is the author/funder, who has granted bioRxiv a license to display the preprint in perpetuity. It is made available under aCC-BY-NC-ND 4.0 International license.

77 (D) Engineering CU-REWIRE to editing M protein (SARS-Cov2). The editing rates of all cytidines nearby the PUF 78 binding site were measured by Sanger sequencing (Left), with the editing rate of the on-target site C246 shown 79 on the right. Values represent mean \pm s.e.m. $(n=3)$. 
A

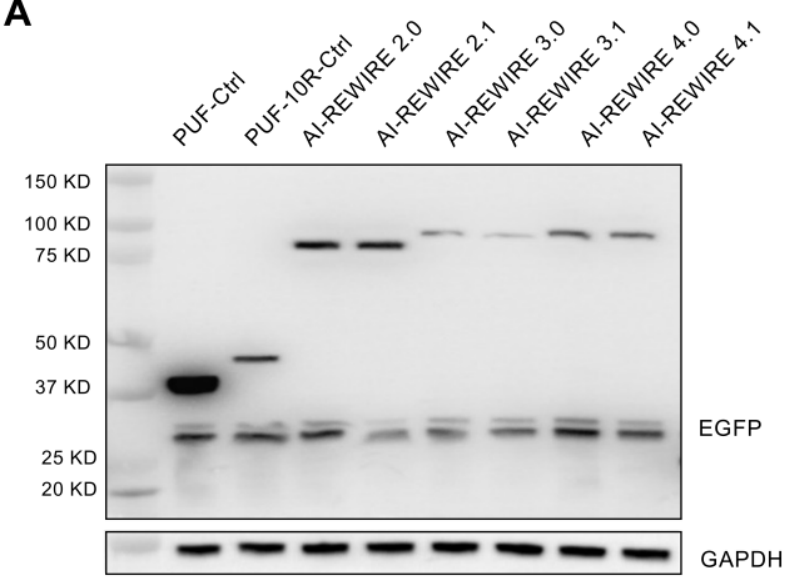

C

Endogenous, PPIB mRNA
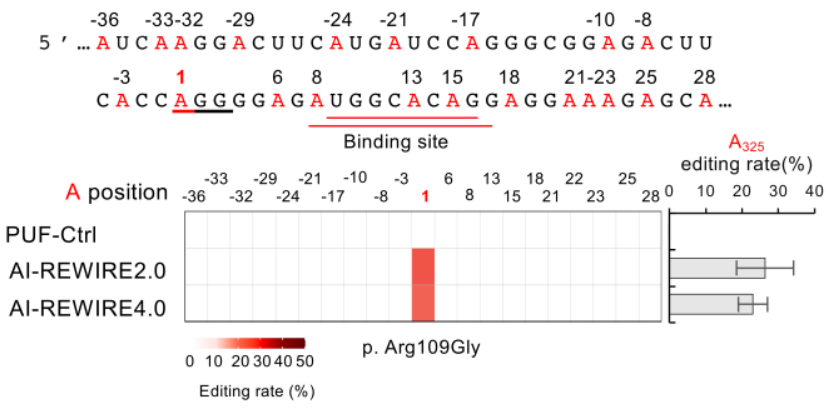

B

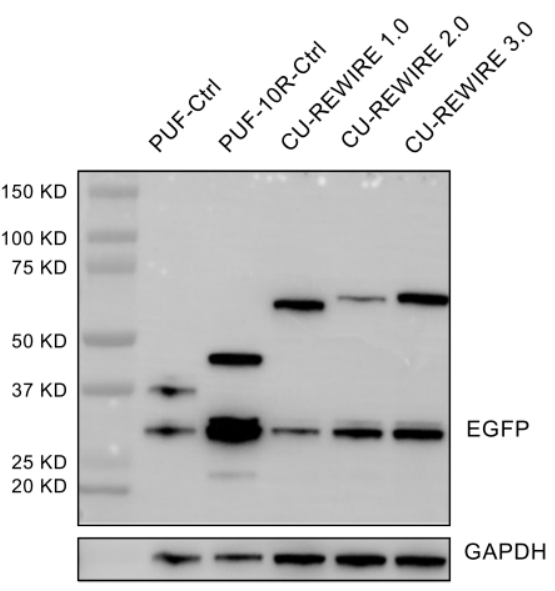

D

E

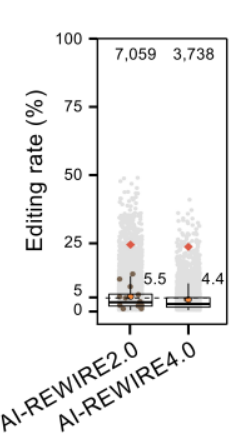

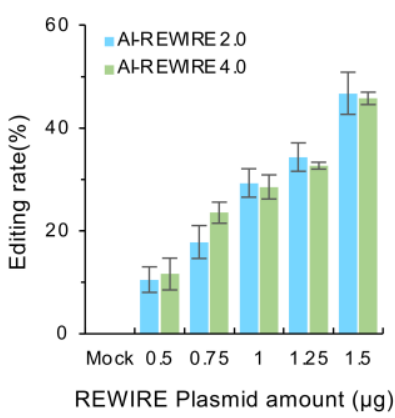

$\mathbf{F}$

Exogenous, EZH2: c.2191T>C (p.Tyr731His) mRNA

Replicate 1

Replicate 2

Replicate 3

$\mathrm{C}>\mathrm{U}$ editing rate $(\%)$
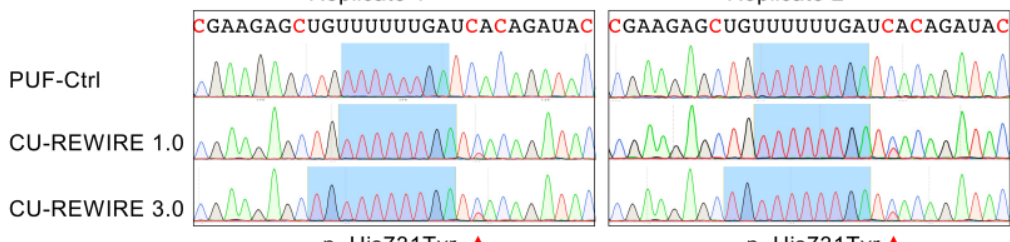

p. His731Tyr
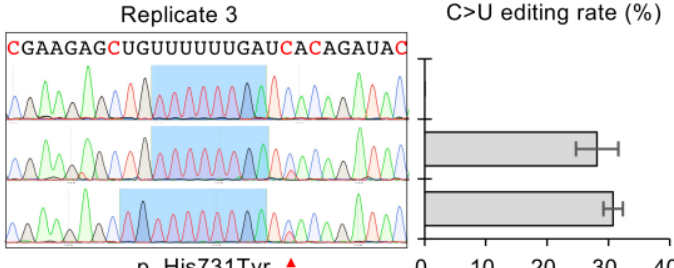

G

Exogenous, SCN1A: c.2588T>C (p. Ser863Leu) mRNA

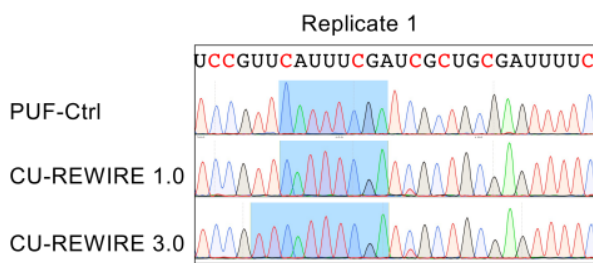

p. Leu863Ser ${ }^{\star}$
Replicate 2

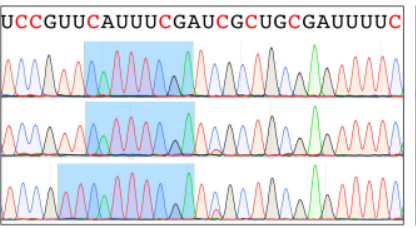

p. Leu863Ser ${ }^{4}$
Replicate 3

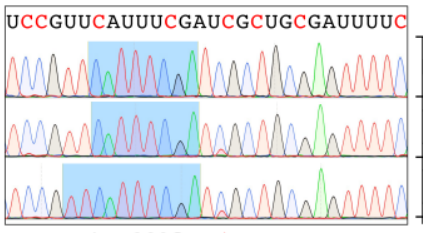

p. Leu863Ser ${ }^{4}$ $\mathrm{C}>\mathrm{U}$ editing rate $(\%)$

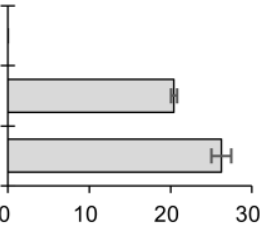

Figure S6. Protein expression level of upgraded REWIREs and assessment on RNA editing efficacy of original and upgraded REWIREs.

(A) Protein expression levels of PUF-control and AI-REWIREs in HEK 293T cells. Representative images of western blot were the same samples in Figure 4B. The western blot was done in technical duplicate. 
(B) Western blot showed the protein expression levels of PUF-control and CU-REWIREs in HEK293T cells. Representative Western blot results used the same samples in Figure 4E. The western blot was done in technical duplicate.

(C) Editing of PPIB mRNA by original and upgraded Al-REWIREs versions in HEK293T cell line. The PUF binding site is underlined in red, with the nearby adenosines marked in red. The editing rates of all adenosines nearby the PUF binding site were measured by RNA-seq (left), with the editing rate of the on-target site A325 shown on the right. Values represent mean \pm s.e.m. $(n=3)$.

(D) Editing rates of transcriptome-wide A-to-I RNA editing in the samples treated with different AI-REWIREs (data from Figure S6C with minimum coverage cutoff at 100). The on-target PPIB site (325 A>G) is highlighted as red rhombuses, average editing rates of off-targets in each sample are indicated with orange dots and values are nearby; the $5 \%$ editing rate was indicated with a dashed line. The editing sites in endogenous genes with an identical PUF binding site sequence in close vicinity (+/- 20nt) were also marked in brown.

(E) Bar plots showing the on-target RNA base editing rates of PPIB mRNA with different doses of original and upgraded Al-REWIREs expression vectors. The editing rates of the A325 site were tested by the Sanger sequencing. Values represent mean \pm s.e.m. $(n=3)$.

(F) Correction of EZH2 2191T >C mutation associated with Weaver syndrome disease by CU-REWIREs. The PUF binding site is highlighted in blue, with the nearby cytidines marked in red. The editing rates of all cytidines nearby the PUF binding site were measured by Sanger sequencing (left), with the editing rate of on-target of C2191 shown in right. Values represent mean \pm s.e.m. $(n=3)$.

(G) Correction of SCN1A 2588T>C mutation by CU-REWIREs. The editing rates of all cytidines nearby the PUF binding site were measured by Sanger sequencing (left), with the editing rate of on-target of C2588 shown in right. Values represent mean \pm s.e.m. $(n=3)$. 

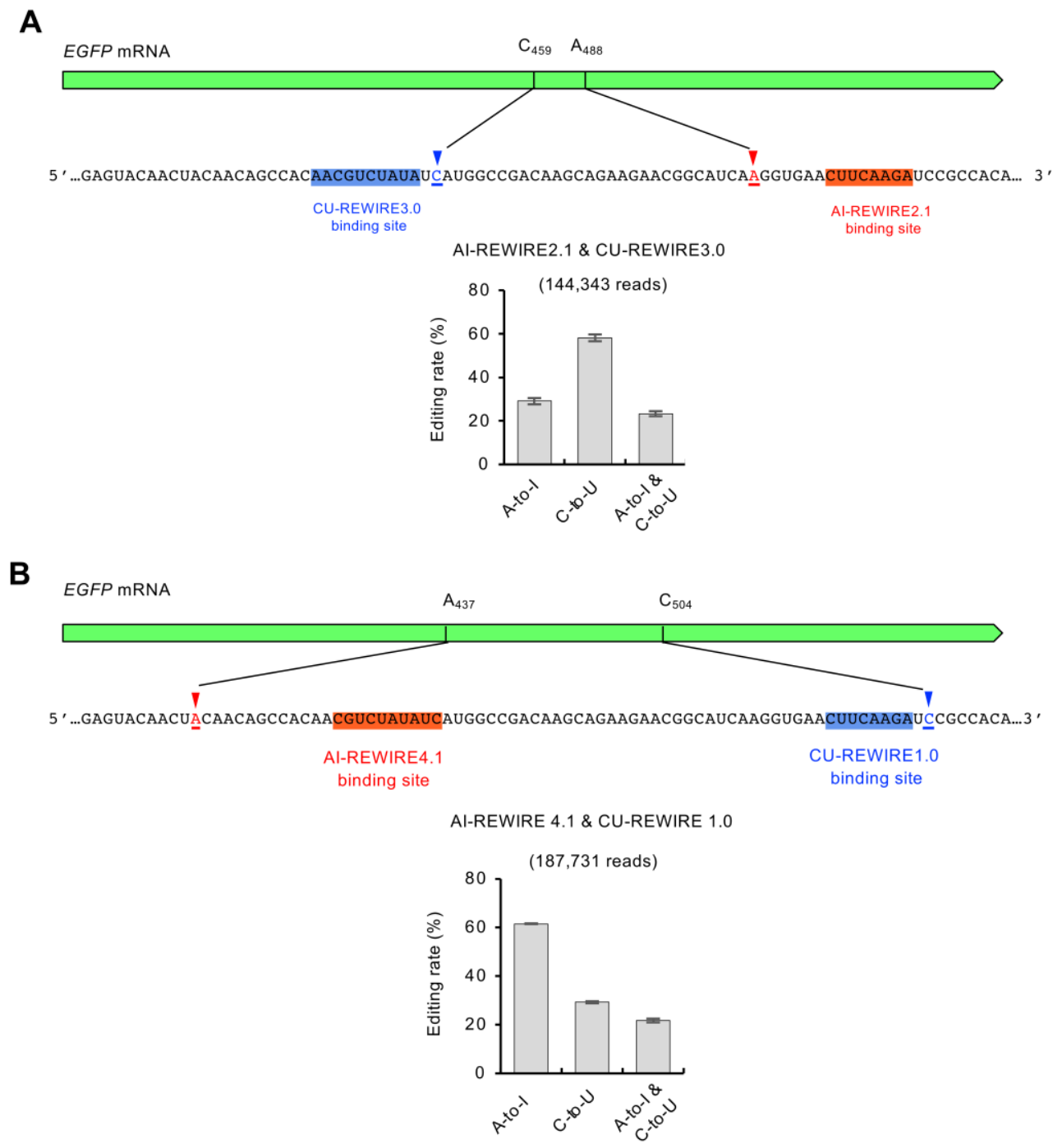

110 Figure S7. RNA base editing coordination mediated by AI\&CU-REWIREs on the same transcript.

111 (A) Schematic diagram of AI\&CU-REWIREs achieved simultaneous base editing in the same transcript (top).

112 Editing rates of the coordination with AI-REWIRE 2.1 and CU-REWIRE 3.0 measured by RNA-seq (bottom).

113 (B) Schematic diagram of AI\&CU-REWIREs targeted to different sites in the same EGFP transcript (top). Editing 114 rates of the coordination with AI-REWIRE 4.1 and CU-REWIRE 1.0 measured by RNA-seq (bottom). 
bioRxiv preprint doi: https://doi.org/10.1101/2021.08.31.458316; this version posted August 31, 2021. The copyright holder for this preprint (which was not certified by peer review) is the author/funder, who has granted bioRxiv a license to display the preprint in perpetuity. It is made available under aCC-BY-NC-ND 4.0 International license.

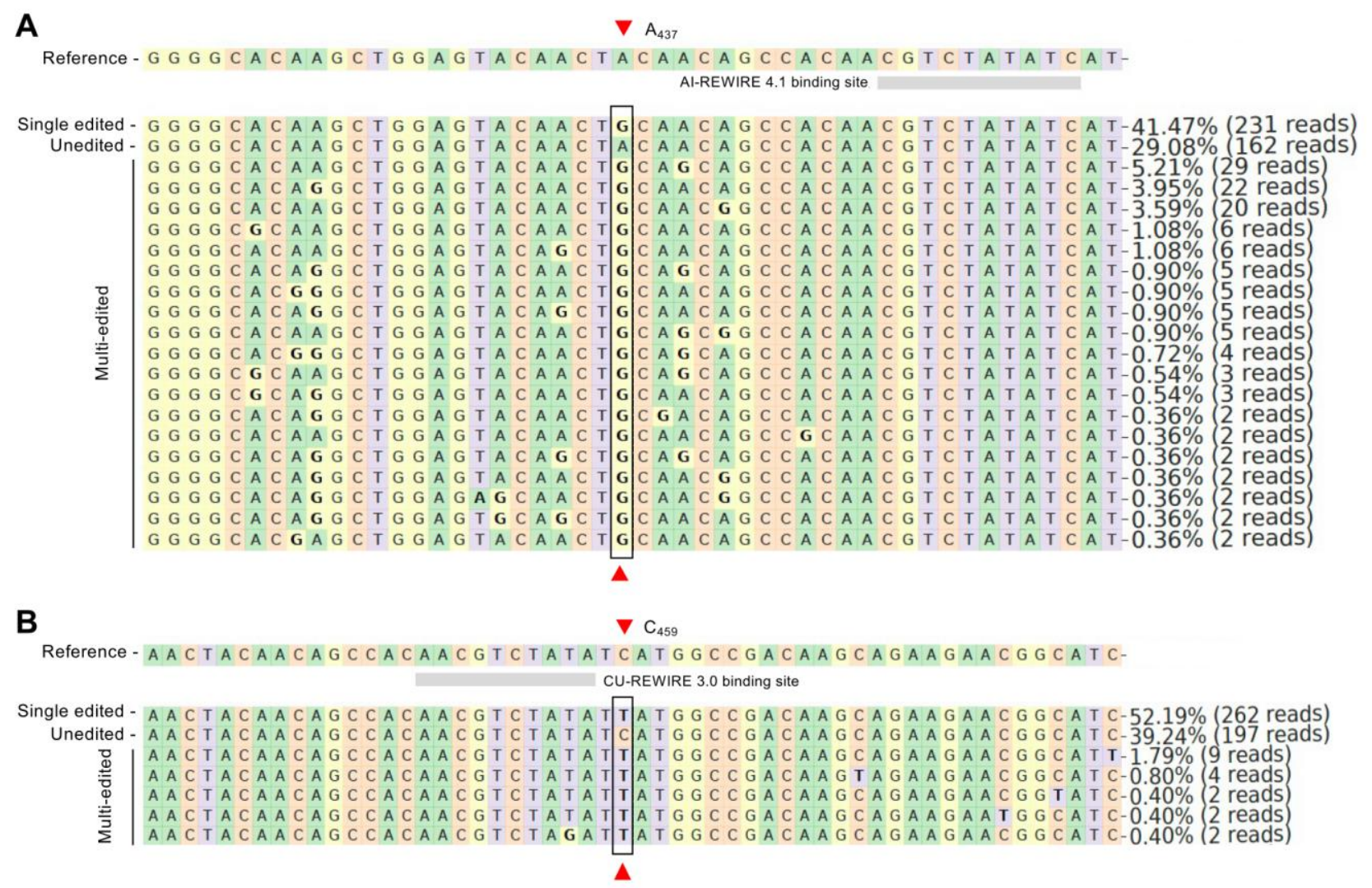

120 Figure S8. RNA editing allele table around target sites on EGFP mRNA by the AI-REWIRE 4.1 (A) and CU121 REWIRE3.0 (B) in 293T cells.

122 The target sites are indicated by red triangle. The PUF binding sites are underlined by grey tile. The percentile and 123 number of reads of each sequence are listed. In this analysis, one representative data of three independent 124 experiments was used; 200-nt window around targeted sites (100-nt upstream and downstream respectively) was 125 used as reference; aligned reads must share $\geqslant 60 \%$ homology with reference sequence in Needleman-Wunsch 126 alignment and have mapping quality $\geqslant 30$; minimal depth cut to plot for one allele is 2. (A) AI-REWIRE4.1 127 replicate1; (B) CU-REIWIRE3.0 replicate1.

128 
A
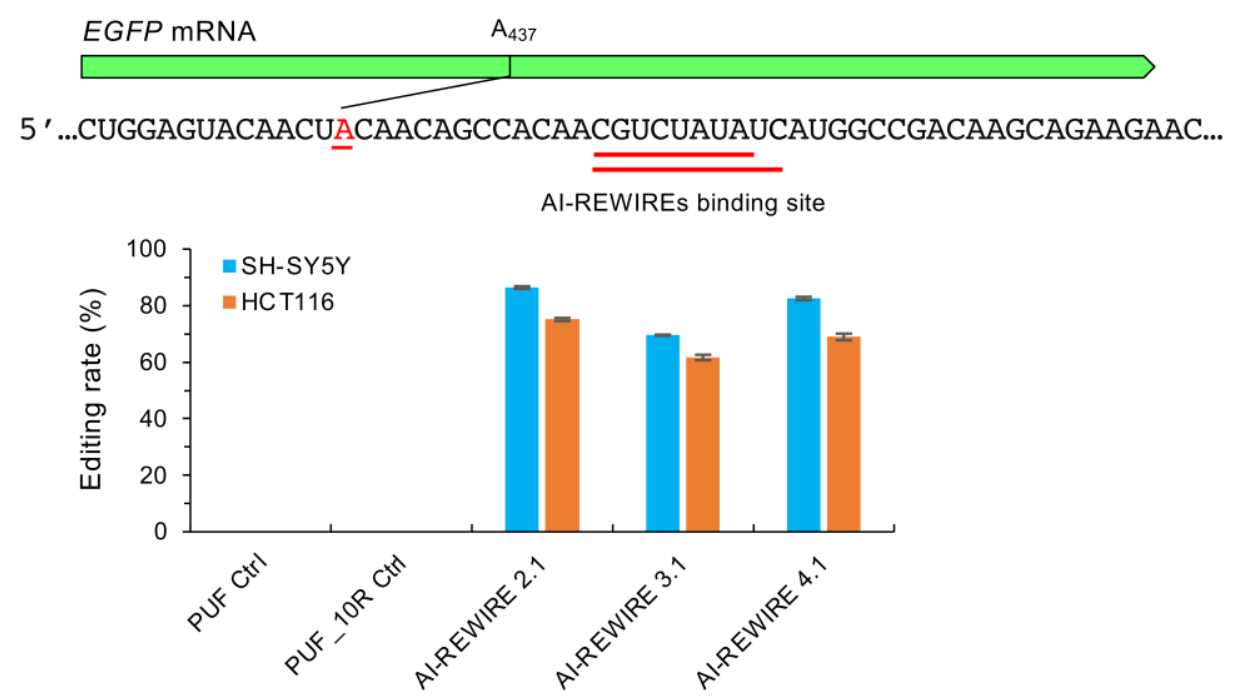

B
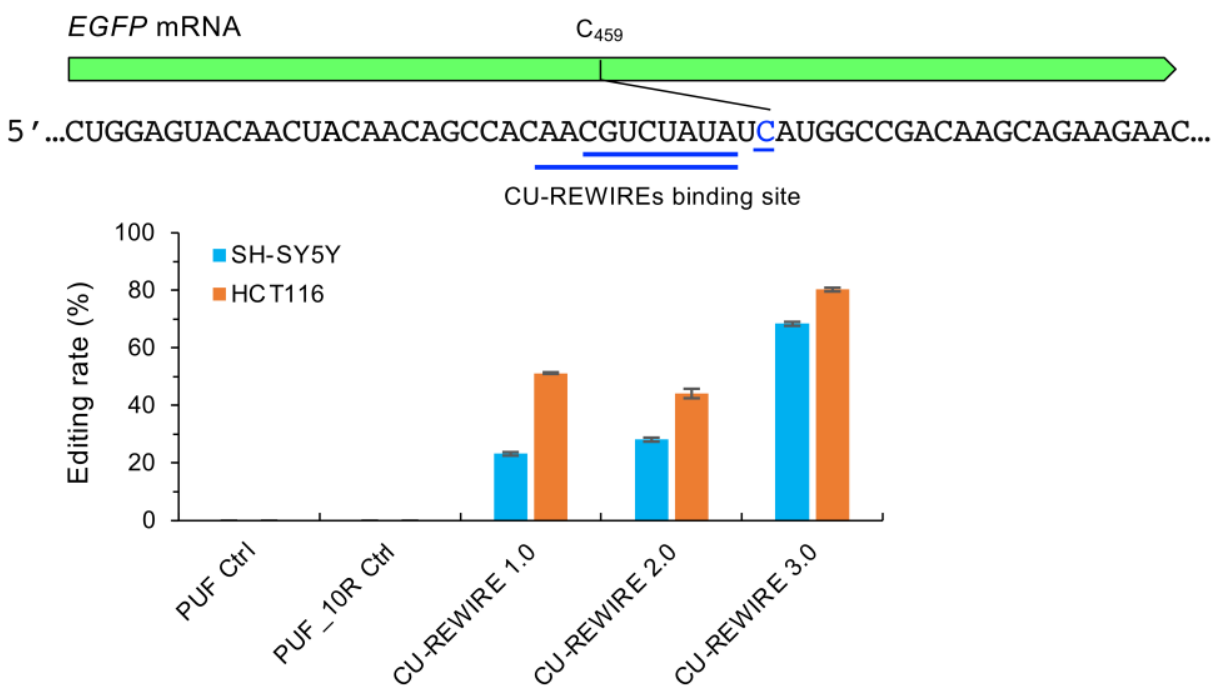

131 Figure S9. Application of REWIREs in other cancer cell lines.

132 (A) Editing of EGFP mRNA by different AI-REWIRE versions in SH-SY5Y and HCT116 cell lines. The binding site of AI-REWIRE 2.1 in EGFP mRNA is underlined in black, AI-REWIRE 3.1/4.1 is underlined in red. The targeted cells were transfected with EGFP reporter and different versions of AI-REWIREs, and the total RNAs were extracted and purified 48 hours later. The editing rate of on-target site A437 was measured with Sanger sequencing $(n=3)$.

(B) Editing of EGFP mRNA by different versions of CU-REWIREs in SH-SY5Y and HCT116 cell line. The binding site of CU-REWIRE 1.0 in EGFP mRNA is underlined in black, CU-REWIRE 2.0/3.0 is underlined in blue. The targeted cells were transfected with EGFP reporter and different versions of CU-REWIREs, and the total RNAs were extracted and purified 48 hours later. The editing rate of on-target site C459 of EGFP mRNA was measured with Sanger sequencing $(n=3)$. 
A

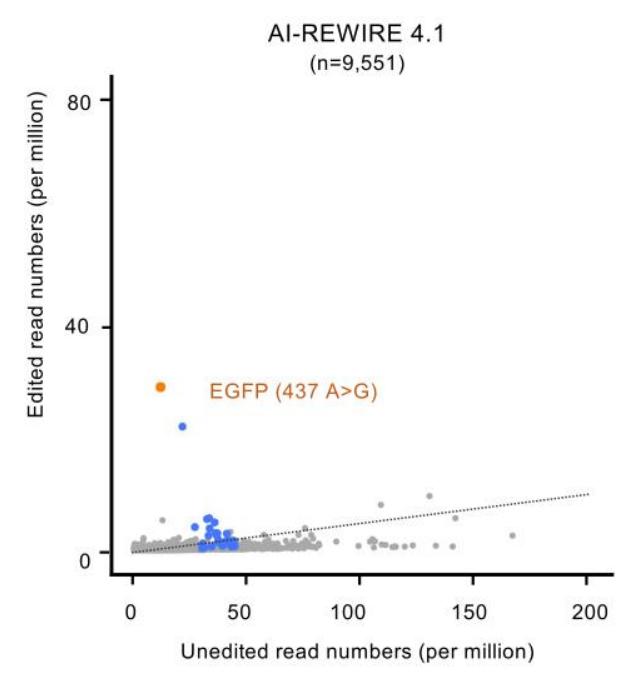

C

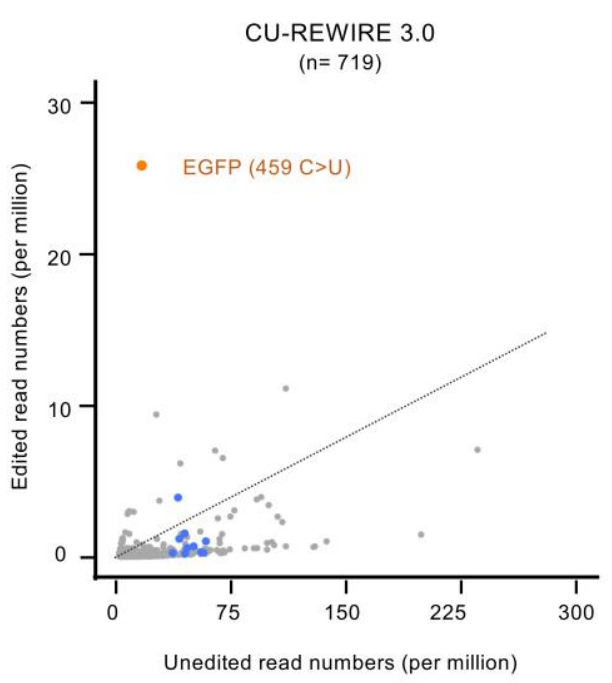

B
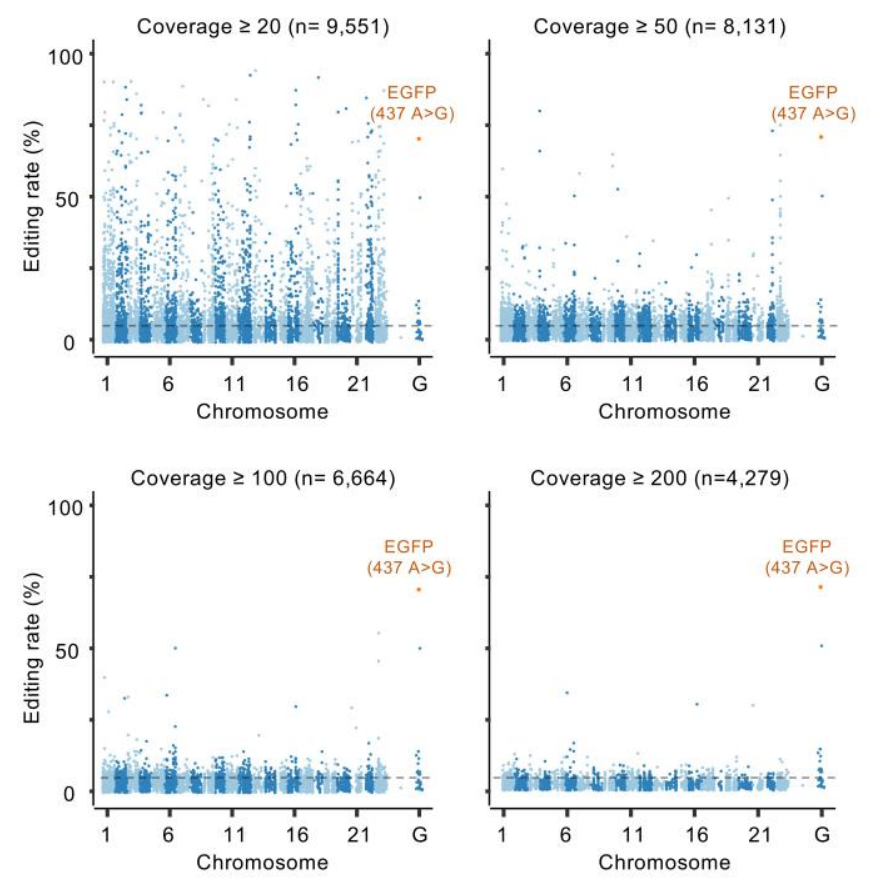

Coverage $\geq 200(n=4,279)$

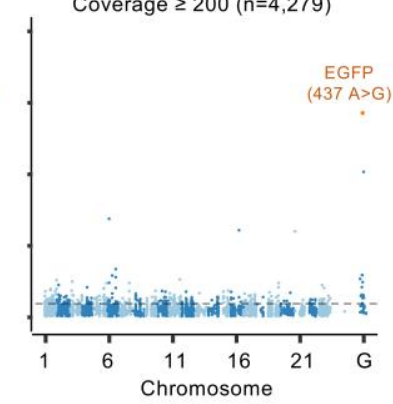

D
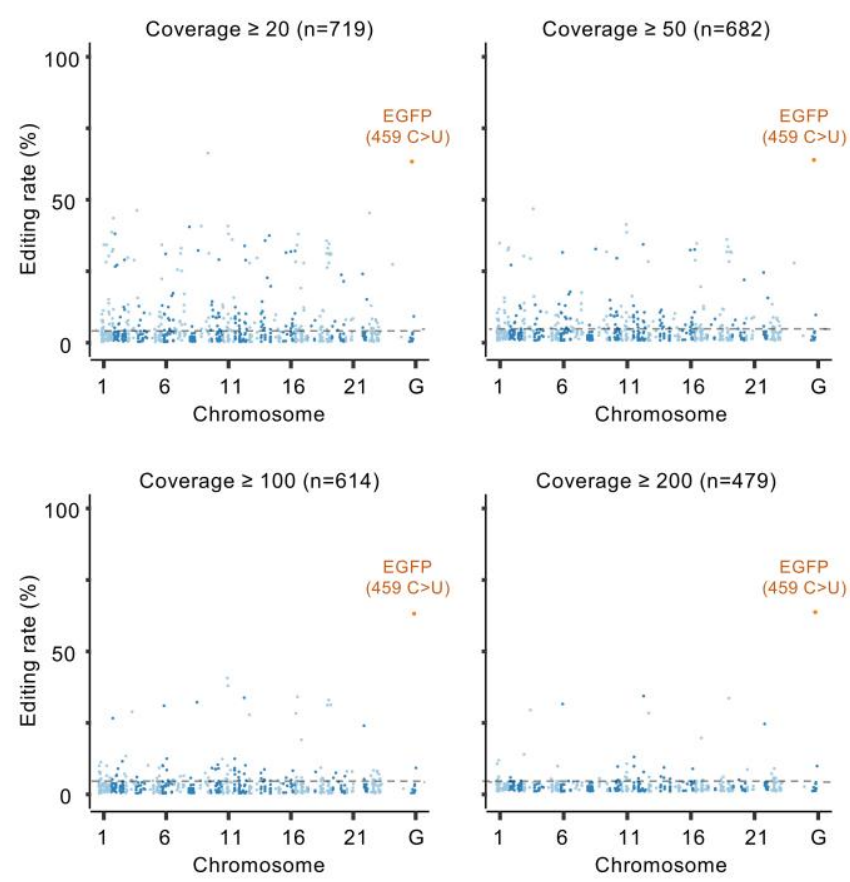
total RNAs from each sample were sequenced using Illumina $\mathrm{Hi}$-Seq $X$ Ten. For each edited adenosine in human transcriptome, the numbers of unedited reads ( $x$-axis) and edited reads ( $y$-axis) were plotted. The experiments and analyses were carried out as described in Figure $1 \mathrm{C}$ with similar labels. 

available under aCC-BY-NC-ND 4.0 International license.

148 (B) The off-target analysis across the entire transcriptome with different coverage thresholds. $n$, numbers of all 149 adenosines detected.

150 (C) Scatter plots of transcriptome-wide C-to-U RNA editing sites in the samples treated with CU-REWIRE3.0. The 151 experiments and analyses were carried out as described in Figure $3 \mathrm{C}$ with similar labels.

152 (D) The off-target analysis across the entire transcriptome with different coverage thresholds. $n$, numbers of all 153 cytosines detected. 